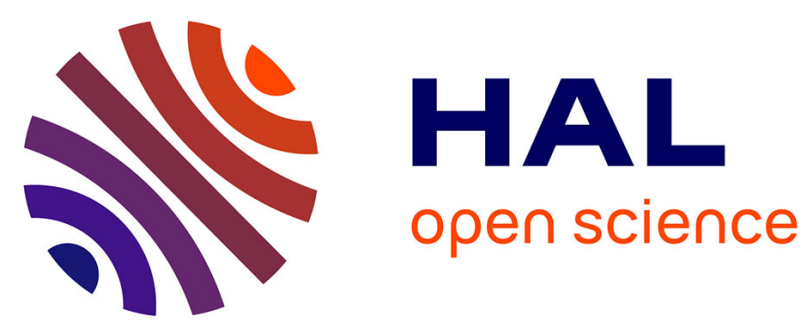

\title{
Genetic variants in autism-related CNTNAP2 impair axonal growth of cortical neurons
}

\author{
Giorgia Canali, Marta Garcia, Bruno Hivert, Delphine Pinatel, Aline
}

Goullancourt, Ksénia Oguievetskaia, Margaux Saint-Martin, Jean-antoine

Girault, Catherine Faivre-Sarrailh, Laurence Goutebroze

\section{To cite this version:}

Giorgia Canali, Marta Garcia, Bruno Hivert, Delphine Pinatel, Aline Goullancourt, et al.. Genetic variants in autism-related CNTNAP2 impair axonal growth of cortical neurons. Human Molecular Genetics, 2018, 27 (11), pp.1941-1954. 10.1093/hmg/ddy102 . hal-01963618

\section{HAL Id: hal-01963618 \\ https://hal-amu.archives-ouvertes.fr/hal-01963618}

Submitted on 21 Dec 2018

HAL is a multi-disciplinary open access archive for the deposit and dissemination of scientific research documents, whether they are published or not. The documents may come from teaching and research institutions in France or abroad, or from public or private research centers.
L'archive ouverte pluridisciplinaire HAL, est destinée au dépôt et à la diffusion de documents scientifiques de niveau recherche, publiés ou non, émanant des établissements d'enseignement et de recherche français ou étrangers, des laboratoires publics ou privés.

\section{(c)(1)}

Distributed under a Creative Commons Attribution| 4.0 International License 


\title{
Genetic variants in autism-related CNTNAP2 impair axonal growth of cortical neurons
}

\author{
Giorgia Canali $^{1,2,3}$, Marta Garcia ${ }^{1,2,3}$, Bruno Hivert ${ }^{4, \dagger}$, Delphine Pinatel ${ }^{4, \ddagger}$, \\ Aline Goullancourt ${ }^{1,2,3}$, Ksenia Oguievetskaia ${ }^{1,2,3}$, Margaux Saint-Martin ${ }^{5}$, \\ Jean-Antoine Girault ${ }^{1,2,3}$, Catherine Faivre-Sarrailh ${ }^{4, \pi}$ and \\ Laurence Goutebroze ${ }^{1,2,3, *}$
}

\author{
${ }^{1}$ Inserm, UMR-S 839, F-75005 Paris, France, ${ }^{2}$ Faculté des Sciences et Ingénierie, Sorbonne Université, F-75005 Paris, \\ France, ${ }^{3}$ Institut du Fer à Moulin, F-75005 Paris, France, ${ }^{4}$ Aix Marseille Université - CNRS, UMR 7286 CRN2M, \\ F-13344 Marseille, France and ${ }^{5}$ CNRS UMR-5310, INSERM U-1217, Institut NeuroMyoGène, Université Claude \\ Bernard Lyon 1, F-69003 Lyon, France \\ *To whom correspondence should be addressed. Tel: + 33 145876144; Fax: + 33 145876132; Email: laurence.goutebroze@inserm.fr
}

\begin{abstract}
The CNTNAP2 gene, coding for the cell adhesion glycoprotein Caspr2, is thought to be one of the major susceptibility genes for autism spectrum disorder (ASD). A large number of rare heterozygous missense CNTNAP2 variants have been identified in ASD patients. However, most of them are inherited from an unaffected parent, questioning their clinical significance. In the present study, we evaluate their impact on neurodevelopmental functions of Caspr2 in a heterozygous genetic background. Performing cortical neuron cultures from mouse embryos, we demonstrate that Caspr2 plays a dose-dependent role in axon growth in vitro. Loss of one Cntnap2 allele is sufficient to elicit axonal growth alteration, revealing a situation that may be relevant for CNTNAP2 heterozygosity in ASD patients. Then, we show that the two ASD variants I869T and G731S, which present impaired binding to Contactin2/TAG-1, do not rescue axonal growth deficits. We find that the variant R1119H leading to protein trafficking defects and retention in the endoplasmic reticulum has a dominant-negative effect on heterozygous Cntnap2 cortical neuron axon growth, through oligomerization with wild-type Caspr2. Finally, we identify an additional variant (N407S) with a dominant-negative effect on axon growth although it is well-localized at the membrane and properly binds to Contactin2. Thus, our data identify a new neurodevelopmental function for Caspr2, the dysregulation of which may contribute to clinical manifestations of ASD, and provide evidence that CNTNAP2 heterozygous missense variants may contribute to pathogenicity in ASD, through selective mechanisms.
\end{abstract}

\section{Introduction}

Autism spectrum disorder (ASD) represents a heterogeneous group of early neurodevelopmental diseases, which are characterized by the presence of repetitive/restricted behaviours and deficits in social interaction and communication (DMS-V). It affects $\sim 1 \%$ of the population, and presents an extremely complex genetic architecture, probably shaped by a

\footnotetext{
'Present address: Institut de Neurosciences de la Timone, UMR 7289, Aix Marseille Université - CNRS, F-13385 Marseille, France. ${ }^{\ddagger}$ Present address: Institut NeuroMyoGène, CNRS UMR-5310, INSERM U-1217, Université Claude Bernard Lyon 1, F-69003 Lyon, France. "Present address: Aix Marseille Université - INSERM UMR1249, INMED, F-13273 Marseille, France. Received: January 27, 2018. Revised: March 14, 2018. Accepted: March 15, 2018 
combination of rare deleterious variants and a myriad of lowrisk alleles (1). Rather than implicating dysfunction in a particular brain structure, ASD is considered to result from abnormal development and functioning of brain connectivity between cortical areas (2-4). Current genetic and neurobiological data indicate that affected stages of development could include prenatal events such as neuronal migration and axon pathfinding, which establish proper positioning and patterning of basal connectivity, and postnatally events of dendritic development and synaptogenesis (5-7).

The CNTNAP2 gene is described as one of the major susceptibility genes for neurodevelopmental disorders, including ASD as well as Gilles de la Tourette syndrome, intellectual disability, obsessive compulsive disorder, cortical dysplasia-focal epilepsy syndrome, schizophrenia, Pitt-Hopkins syndrome and attention deficit hyperactivity disorder (8-10). It encodes the neuronal neurexin-like cell adhesion transmembrane glycoprotein Contactin-associated protein-2 (Caspr2), which is well-known for its role in axo-glial contacts of matured myelinated axons, forming a complex in cis and in trans with the glycosylphosphatidylinositol (GPI)-anchored immunoglobulin cell-adhesion molecule CNTN2/Contactin2/TAG-1 at the juxtaparanodal regions of the nodes of Ranvier (11-13). Caspr2 is required for the clustering of Shaker-type voltage-gated potassium channels (Kv1.1 and Kv1.2) in these regions $(11,14)$. Mislocalization of Kv1 channels in Cntnap $2^{-1-}$ mice is associated with modifications of axonal action potential wave form and increases in postsynaptic excitatory responses (14), suggesting that Caspr2 is important for proper conductivity of central myelinated fibers. Recent data provided evidence that Caspr2 may play several other roles during early post-natal neurodevelopment and contribute to normal neuronal network assembly and activity. Caspr2 was found to be important for the maturation of the parvalbuminpositive $\left(\mathrm{PV}^{+}\right)$GABAergic cortical interneurons and the physiology of fast-spiking $\mathrm{PV}^{+}$neurons $(15,16)$. Knockout and knockdown studies showed that it is required for the normal development of cortical neuron dendritic arborization and spines, synaptic strength and AMPA receptors trafficking (17-19). In addition, a delay in myelination was recently described in juvenile Cntnap $2^{-1-}$ mice (14), the mechanisms of which are not clear, but suggesting that Caspr2 may influence network dynamics of cortical neurons.

CNTNAP2 genetic alterations identified in ASD patients include complex genomic rearrangements, heterozygous intragenic deletions and especially a large number of rare heterozygous missense variants distributed over the entire extracellular domain of Caspr2 $(8,10,20,21)$. It has been proposed that CNTNAP2 intragenic deletions may exert a dominant-negative effect, leading to expression of Caspr2 proteins deleted of part of their extracellular domains, which may perturb the normal functions of Caspr2 (22). Most of the heterozygous missense variants are inherited from an unaffected parent of the ASD patients (21). Also, heterozygous missense CNTNAP2 variants have been found in control individuals (20), thus questioning the clinical significance of the variants identified in ASD patients, and therefore their contribution to the development of the pathology. So far, the impact of the variants on the functions of Caspr2 has been poorly evaluated because of lack of reliable assay. The extracellular domain of Caspr2 is composed of different structural subdomains and is likely to present an overall compact architecture, suggesting complex structurefunction relationships $(23,24)$. Studies in HEK-293 transfected cells showed that some variants are misfolded and present severe trafficking abnormalities, being largely retained in the endoplasmic reticulum (ER), while others are well localized at the plasma membrane (25). However, the functional consequences of the misfolded variants have not been further evaluated. In addition, four variants localized at the plasma membrane were shown to act like null/hypomorphic alleles based on their inability to rescue the maturation of Cntnap2 $2^{-1-} \mathrm{PV}^{+}$cortical interneurons in a transplantation assay (15), but the causes of the phenotype are not clear.

Here, we aimed at developing biochemical approaches and a sensitive developmental in vitro cell assay to clarify the potential functional impact of various CNTNAP2 missense variants in a heterozygous Cntnap2 background relevant for CNTNAP2 heterozygosity in ASD patients. The expression pattern of Cntnap2 in mouse suggested a role for Caspr 2 in cortical neurons as early as embryonic day 14 (E14) (16). We performed neuronal cultures from wild-type, Cntnap2 $2^{+-}$and Cntnap $2^{-/-}$embryos at E14.5, and showed that Caspr2 regulates axonal outgrowth in a dosedependent manner. Testing variants in Cntnap $2^{+/-}$neurons, we found that they have different effects on axon elongation, likely through different mechanisms, thus providing a proof of principle that certain CNTNAP2 heterozygous missense variants may contribute to ASD pathogenicity.

\section{Results}

\section{Caspr2 modulates axonal outgrowth of primary E14.5 cortical neurons in a dose-dependent manner}

Dissociated E14.5 cortical neurons were first cultured from wildtype (WT) embryos. RT-PCR and immunoblotting experiments showed that these neurons expressed Cntnap2/Caspr2 at 3 and 7 days in culture (DIV3 and DIV7) (Fig. 1A and B). Cell surface immunolabelling with purified anti-Caspr2 antibodies from a patient affected by an autoimmune limbic encephalitis (26) demonstrated a faint staining on the soma and along the neurites, including the axon, and at the level of the growth cones (Fig. 1C, left panels). This staining, which was not detectable in neuronal cultures from Cntnap2 ${ }^{-/-}$mutant (KO) neurons (Fig. 1C, right panels), suggested that Caspr2 could contribute to neuronal neuritogenesis. We thus analyzed neuronal morphology in cultures generated from WT, Cntnap2 ${ }^{+/-}$(HET), and KO embryos. Cultures were fixed at DIV2 and DIV3, and measurements were performed on neurons that exhibited one longer Tuj-1 immunopositive process (axon) and several shorter neurites (not shown). A significantly decreased axon length was observed in mutant neurons both at DIV2 and DIV3 (Fig. 1D). Interestingly, HET neurons displayed an intermediate axon length as compared to those of WT and KO neurons. Axonal defects were rescued by electroporating the neurons before plating with a pCAGGS-IRES construct encoding HA-tagged Caspr2 and the fluorescent protein Tomato to detect transfected cells (Fig. 1E). Axon length of HA-Caspr2-expressing neurons was not significantly different between the three genotypes and also remarkably similar to that from WT neurons electroporated with the control vector. These data indicate a role for Caspr2 in axon elongation, which is tightly regulated and dependent on the expression level of the protein.

\section{Caspr2 oligomerizes during its maturation}

The dose-dependent role of Caspr2 in axon growth offered a reliable and sensitive assay to test the impact of ASD-related missense CNTNAP2 variations, and further suggested diverse scenarios by which they could interfere with the normal cellular 
A

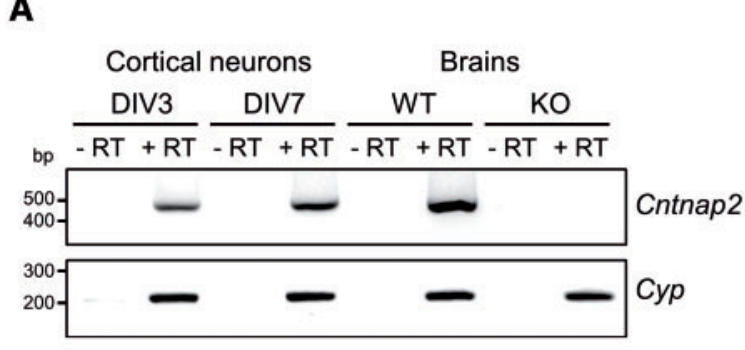

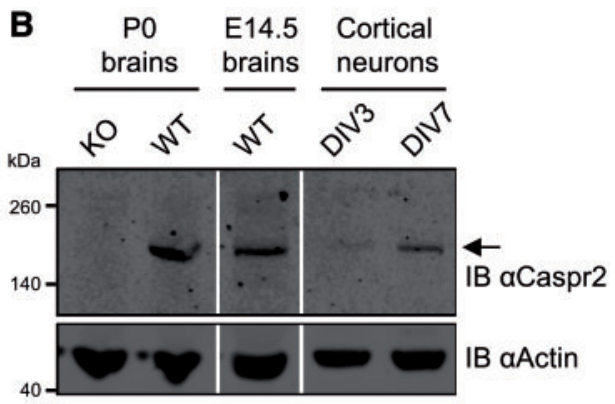

\section{C}
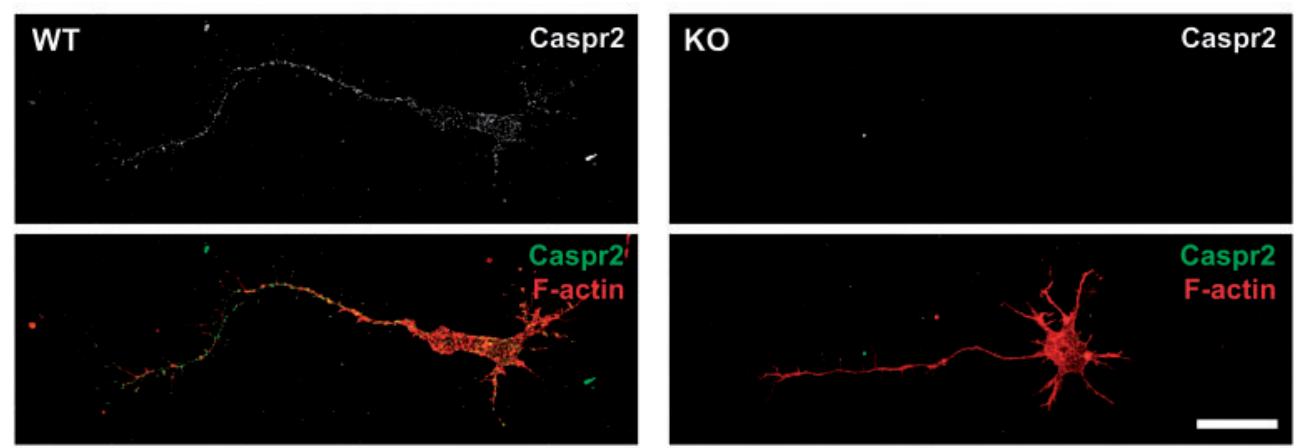

D

E

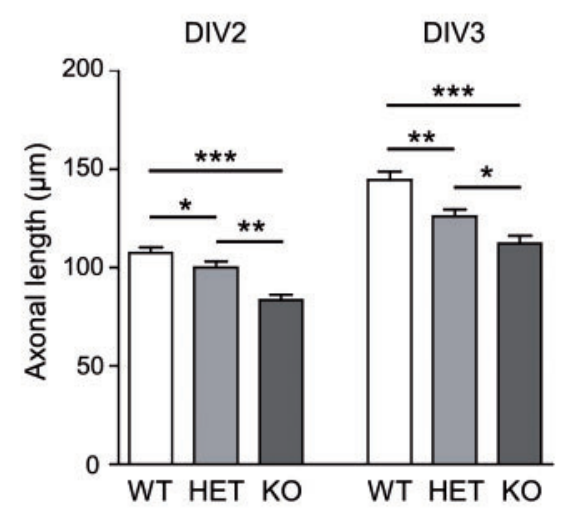

Figure 1. Caspr2 modulates axonal outgrowth of primary E14.5 cortical neuron in a dose-dependent manner. (A) Cntnap2 mRNA levels analysis by RT/PCR on total mRNAs from E14.5 cortical neurons at DIV3 and DIV7, and WT and KO adult mouse brains. Cyp, Cyclophilin B as control. Molecular markers are shown in base pairs (bp) on the left of the panels. Expected size of DNA fragments: Cntnap2, 476 bp; Cyp, 215 bp. (B) Caspr2 protein levels analysis by immunoblotting (IB) on lysates from E14.5 cortical neurons at DIV3 and DIV7, WT and KO newborn (P0) mouse brains, and WT E14.5 brains. Actin was used to normalize protein expression. Molecular mass markers positions are shown in $\mathrm{kDa}$ on the left of the panels. The arrow indicates the band for Caspr2. (C) Representative images of Caspr2 cell surface immunostaining on WT and KO cortical neurons at DIV3 (green). Co-staining of actin with phalloidin (red). (D) Quantification of axon length in WT, HET and KO cortical neurons at DIV2 and DIV3. (E) Rescue of axon growth by expression of HA-Caspr2 in cortical neurons. Quantification of axon lengths at DIV3 in WT, HET and KO neurons electroporated with the control vector or the HA-Caspr2-expressing vector. (D, E) Data are means + SEM. (D) $n=7-9$ embryos/genotype, $n=33-36$ neurons/embryo. (E) $n=8-10$ embryos/genotype, $n=40-43$ neurons/embryo. Statistical analyses: Kruskal-Wallis one-way ANOVA test, (D, E) $P<0.0001$; Dunn's Multiple Comparison post-test, ${ }^{*} \mathrm{P}<0.05,{ }^{* *} \mathrm{P}<0.01,{ }^{* * *} \mathrm{P}<0.001$; ns, not significant. (C) Scale bar, $25 \mu \mathrm{m}$.

functions of Caspr2 on a heterozygous CNTNAP2 background. One scenario was that Caspr2 variants could interact with WT Caspr2, impair its trafficking, subcellular localization and/or function at the membrane, leading to a homozygous cellular phenotype. This was suggested by the fact that oligomerization in ER is a quite general mechanism used by biosynthetic quality-control checkpoints to make sure that only properly folded membrane glycoproteins reach their site of action. Thus, in preliminary variant studies, we evaluated this possibility and asked whether WT Caspr2 could oligomerize by performing coimmunoprecipitation from lysates of heterologous cells co- expressing HA-Caspr2 and Myc-tagged Caspr2 (Myc-Caspr2) (Fig. 2A). When expressed in COS7 cells, Caspr2 appeared in immunoblotting as a doublet of two protein bands (Fig. 2B, left panel). Surface biotinylation (Fig. 2B) and digestions with endoglycosidases $\mathrm{H}$ and $\mathrm{F}$ (Fig. 2C) indicated that the upper band corresponds to a mature, glycosylated, transport-competent form that is present at the plasma membrane, while the lower band is an immature glycosylated intracellular form. The mature form of Caspr2 at the cell surface contains complex N-glycans, which are resistant to endoglycosidase $\mathrm{H}$, whereas the immature form of Caspr2 in the ER bears high-mannose N-glycans, 
which are sensitive to endoglycosidase $\mathrm{H}$. In cells co-expressing HA-Caspr2 and Myc-Caspr2, HA antibodies pulled down the immature form of Myc-Caspr2 (Fig. 2D, arrows). When cells were treated for $18 \mathrm{~h}$ before co-immunoprecipitation with tunicamycin, which blocks the first enzyme of the glycosylation pathway and thus inhibits the synthesis of all N-linked oligosaccharides in cells, HA antibodies also pulled down the nonglycosylated form of Myc-Caspr2 (Fig. 2E, arrowheads). These observations demonstrate the ability of Caspr2 to self-associate during its processing in the ER but likely not at the plasma membrane. Further experiments with HA-Caspr2 proteins encompassing sequential deletions of the extracellular domain (Fig. 2A) showed that co-immunoprecipitation of Myc-Caspr2 was dramatically altered by the deletion of the fibrinogen domain and the third laminin $G$ domain (HA-Caspr2 $\Delta 3$, Fig. $2 F$ ), indicating that Caspr2 oligomerization requires at least one of these two domains.

\section{The variants $\mathrm{I} 869 \mathrm{~T}, \mathrm{R} 1119 \mathrm{H}$ and $\mathrm{D} 1129 \mathrm{H}$ oligomerize with WT Caspr2 and have a dominant-negative effect on its subcellular localization in transfected COS7 cells}

Since some Caspr2 variants were previously shown to be misfolded and retained in the ER (25), we next asked whether they could form heteromers with WT Caspr2, and therefore impact its trafficking and subcellular localization. We first screened for variants that present trafficking abnormalities among variants previously reported by Bakkaloglu et al. (21) (Supplementary Material, Fig. S1A). For this, HA-tagged Caspr2 variants were expressed in COS-7 cells and immunoblotting was performed to evaluate the proportion of the immature glycosylated form for each of them (Supplementary Material, Fig. S1B and C). Two variants, HA-R1119H and HA-D1129H, appeared to be mainly expressed as the immature form when compared to WT Caspr2 (Supplementary Material, Fig. S1B and C). ER retention of these variants was further monitored by quantifying the proportion of protein co-localizing with an ER marker (KDEL) in transfected cells (Supplementary Material, Fig. S2A-C). Consistent with previous observations (25), a large proportion of HA-R1119H and HA-D1129H was significantly retained in the ER as compared to WT Caspr2 (Fig. 3B), the retention being higher for HA-D1129H. A third variant, HA-I869T, also appeared largely as an immature form on immunoblotting (Supplementary Material, Fig. S1B and C). Immunostaining showed that its retention in the ER was higher but not significantly different from that of WT Caspr2 (Fig. 3B; Supplementary Material, Fig. S2A and D). We nevertheless considered pursuing its characterization because it had been found in four different ASD families $(20,21)$.

Co-immunoprecipitation experiments on lysates of COS-7 cells co-expressing Myc-Caspr2 demonstrated that the three variants I869T, R1129H and D1129H, were able to form heteromers with WT Caspr2 (Fig. 3C). The proportion of immature form of Myc-Caspr2 co-immunoprecipitated with the variants was remarkably higher than that pulled down by HA-Caspr2, suggesting a stronger association of WT Caspr2 with the variants than with the WT molecules. Immunostaining confirmed that the three variants were retained in the ER in co-transfected cells similarly as when they were expressed alone, although in this case ER retention of I869T appeared significantly different from that of WT Caspr2 (Fig. 3D). The ability of the variants to induce ER retention of WT Caspr2 was monitored by quantifying the proportion of Myc-Caspr2 co-localizing with the ER marker (Supplementary Material, Fig. S3A-D). The retention of
Myc-Caspr2 in the ER was increased in cells co-expressing any of the three variants as compared to cells expressing HA-Caspr2 (Fig. 3E), indicating that these variants have a dominant effect on the subcellular localization of WT Caspr2 in heterologous cells.

\section{The variant $\mathrm{R} 1119 \mathrm{H}$ has a dominant-negative effect on axon growth while the variant I869T does not fully res- cue axon growth defects of HET neurons}

To evaluate the impact of the variants on axonal growth, cortical neurons were electroporated before plating with pCAGGSIRES-Tomato constructs encoding the HA-tagged proteins and fixed at DIV3. Consistent with observations in COS-7 cells, cell surface immunostaining showed that HA-R1119H and HAD1129H were poorly expressed at the surface of HET neurons as compared to WT Caspr2 (Fig. 4A-C). Axon length was measured for $\mathrm{R} 1119 \mathrm{H}$, assumed to be representative of these two variants with similar properties, in neurons of the three genotypes (WT, HET and KO) to make sure to detect any minor effect. A significant decrease in axon growth was observed in both WT and HET neurons expressing HA-R1119H as compared to WT and HET neurons electroporated with the control pCAGGS-IRESTomato vector or expressing HA-Caspr2 (Fig. 4E). In addition, axon growth of HA-R1119H-expressing WT and HET neurons were comparable and not significantly different from that of KO neurons electroporated with the control vector, demonstrating that HA-R1119H has a dominant-negative effect on the role of WT Caspr2 in axonal outgrowth, likely through oligomerization and retention in the ER. In contrast, HA-I869T was detectable at the surface of the neurons as well as WT Caspr2 (Fig. 4A and D). In addition, axon growth of HA-I869T-expressing HET neurons was not decreased but showed an intermediate phenotype between neurons electroporated with the control vector and neurons expressing exogenous WT Caspr2 (Fig. 4F). This indicates that this variant does not play a dominant effect on WT Caspr2 but does not either fully rescue axon growth defects of HET neurons as compared to wild-type Caspr2, and therefore suggests that its structural changes can be overcome in cortical neurons to allow its trafficking, but nevertheless impair its functions at the plasma membrane.

\section{The cell adhesion-defective variant HA-G731S displays loss of function in axonal outgrowth}

One of the most likely causes for the loss of function of the variant $1869 \mathrm{~T}$ could be that its structural changes affect the cell adhesion properties of the protein. To test this hypothesis, as well as to evaluate whether other CNTNAP2 variations could lead to similar functional impairments, we took advantage that Caspr2 was previously shown to interact in trans with the cell-adhesion molecule CNTN2/Contactin2/TAG-1 (26) and asked whether CNTNAP2 variants could impair this interaction by testing the ability of Caspr2-Fc chimera to bind to N2a cells expressing GFP-tagged TAG-1. Besides I869T, we focused on the five variants N407S, N418D, Y716C, G731S and R906H, which did not appear to present major trafficking defects evaluated by immunoblotting (Supplementary Material, Fig. S1A-C). WT or variant Caspr2-FC plasmids were transfected in HEK cells and the recombinant proteins were purified from the culture supernatant using Protein A-affinity chromatography. Same amounts of WT or variant chimera were pre-clustered with fluorescent anti-Fc IgGs and incubated on transfected N2a cells. As previously observed (26), 
A

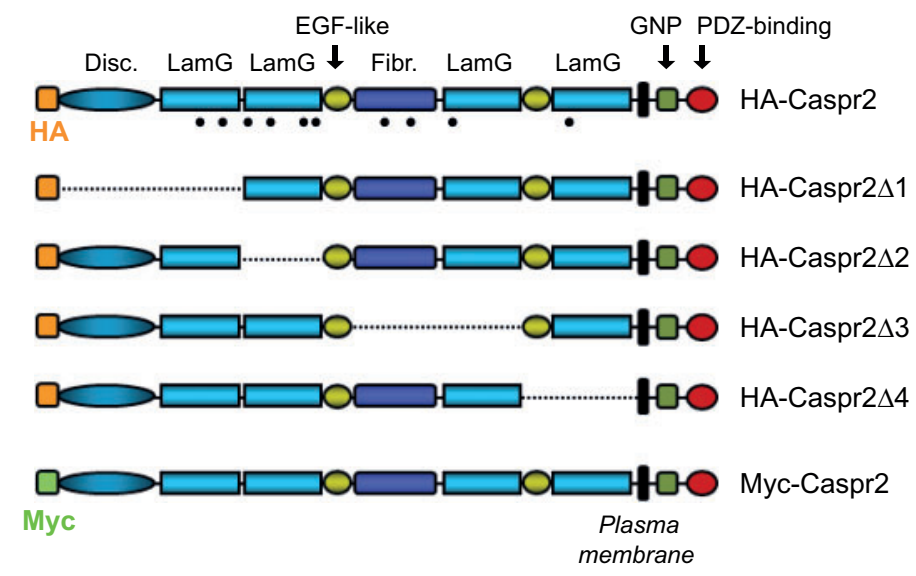

B
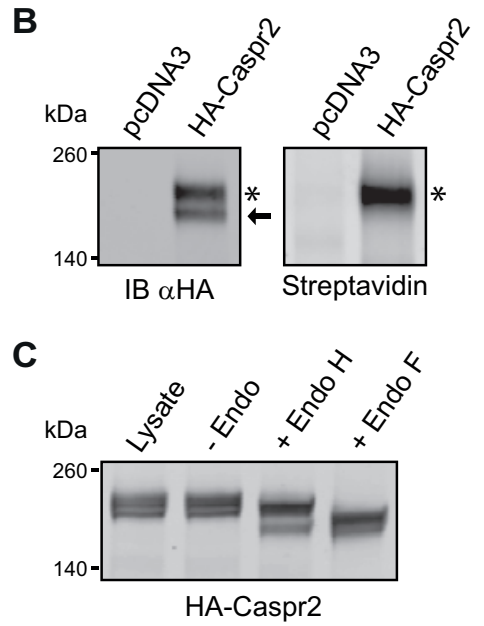

D

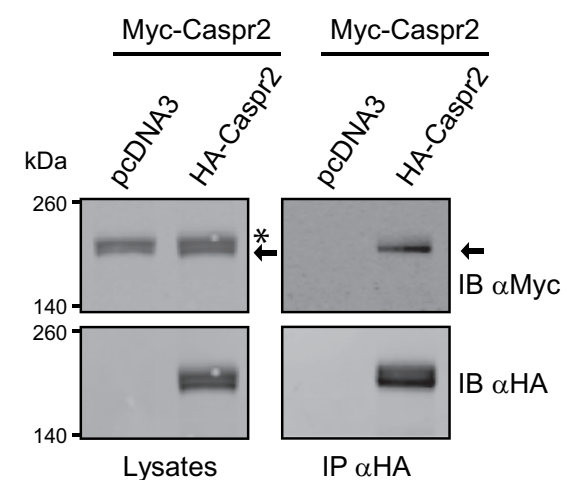

E

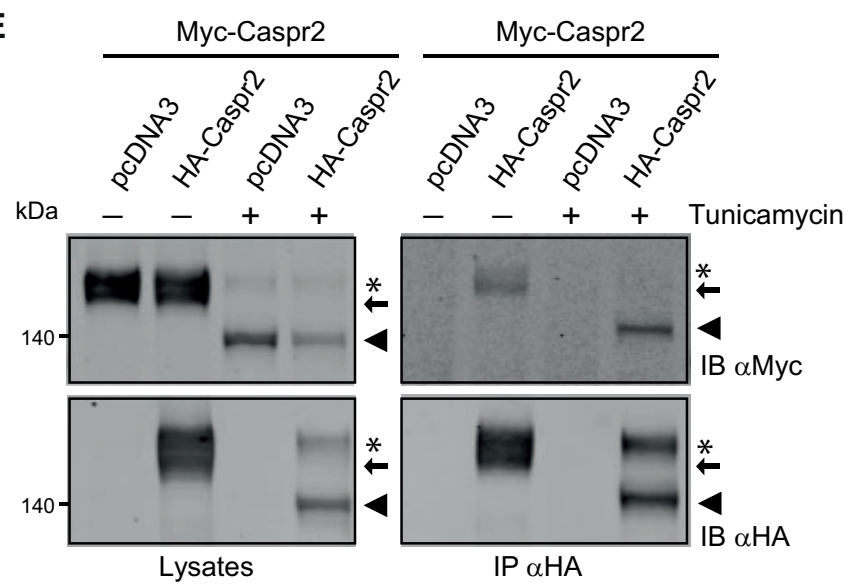

F

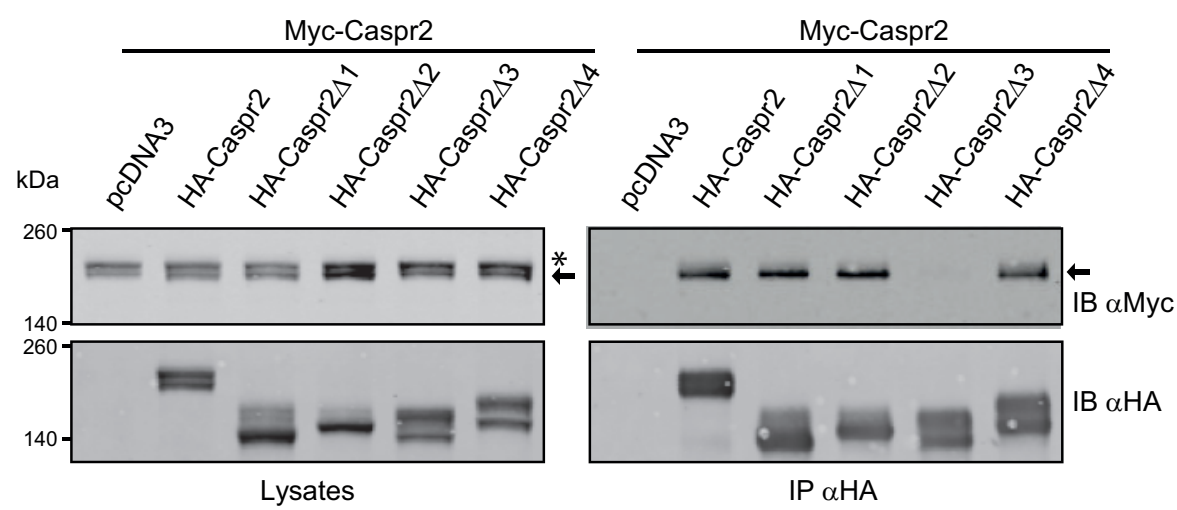

Figure 2. Caspr2 oligomerizes during its maturation. (A) Schematic domain organization of HA-Caspr2, HA-tagged deleted Caspr2 and Myc-Caspr2. Disc., discoidin; LamG, laminin G; Fibr., fibrinogen; EGF-like, EGF-like; GNP, Glycophorin C-Neurexin IV-Paranodin; PDZ-binding, PDZ binding domain. Dashed lines substitute for deleted domains. Black dots indicate the positions of the N-glycosylation sites. (B) Cell surface biotinylation experiment in COS-7 expressing HA-Caspr2 revealed by blotting with HA antibodies (IB $\alpha \mathrm{HA}$ ) and streptavidin. The asterisk indicates the mature biotinylated form of HA-Caspr2 and the arrow the immature intracellular form. (C) Deglycosylation profiles of HA-Caspr2 digested by endoglycosidase H (Endo H) and endoglycosidase F (Endo F). (D, E) Immunoprecipitations (IP) with HA antibodies on lysates from transfected COS-7 cells co-expressing Myc-Caspr2 and HA-Caspr2, non-treated (D) or treated for $18 \mathrm{~h}$ with tunicamycin or DMSO as control (E), and revealed by immunoblotting (IB) with Myc or HA antibodies. Crude protein extracts (Lysates) were immunoblotted to verify protein expression. HA antibodies immunoprecipitate the immature form of Myc-Caspr2 in non-treated cells (D), and the non-glycosylated form of Myc-Caspr2 in lysates from tunicamycin-treated cells (E). (D, E) The asterisks indicate the mature forms of Caspr2, the arrows the immature forms, and the arrowheads the non-glycosylated forms Caspr2. (F) IP with HA antibodies from lysates of transfected COS-7 cells co-expressing Myc-Caspr2 and HA-Caspr2 or HA-tagged Caspr2 deleted of different subdomains of the extracellular domain (HA-Caspr2 $\Delta 1-\Delta 4$ ) revealed with Myc or HA antibodies. Deletion of the fibrinogen domain and the third laminin G domain (HA-Caspr2 $\Delta 3$ ) dramatically altered co-immunoprecipitation of the immature form of Myc-Caspr2. The asterisks indicate the mature forms of Caspr 2 and the arrows the immature forms. Molecular mass markers positions are shown in $\mathrm{kDa}$ on the left of the panels. 
A

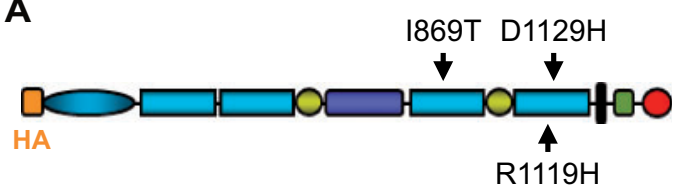

B

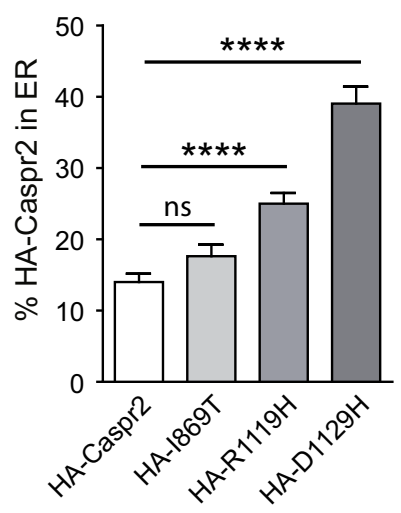

D

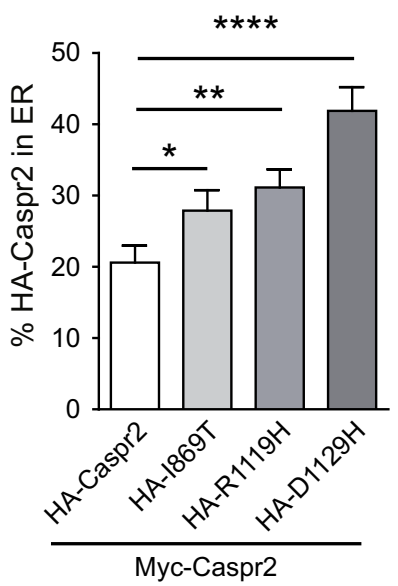

C
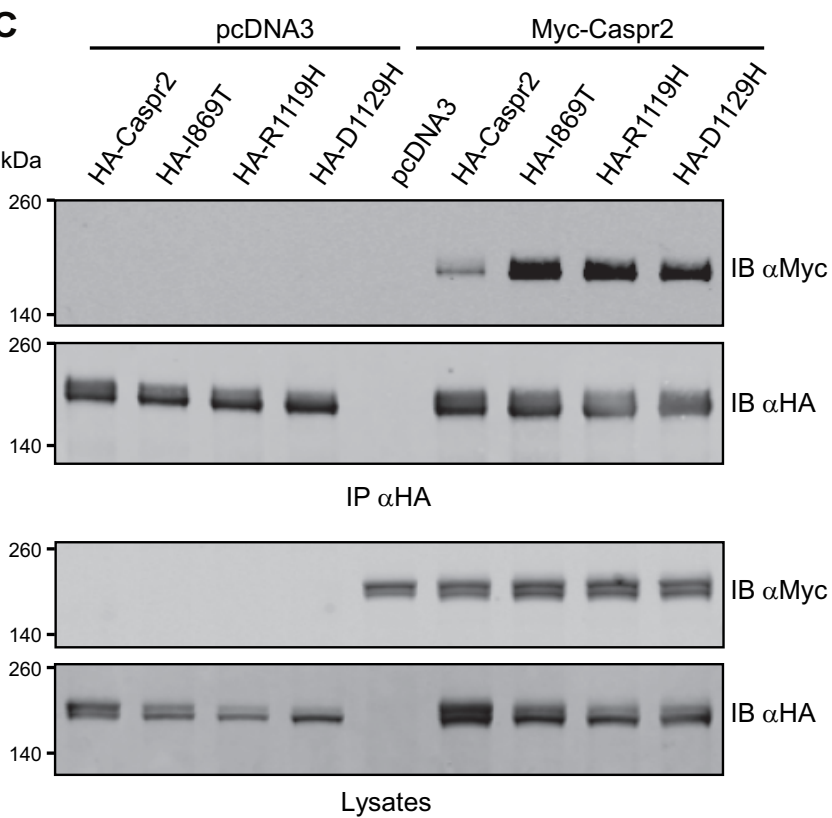

E

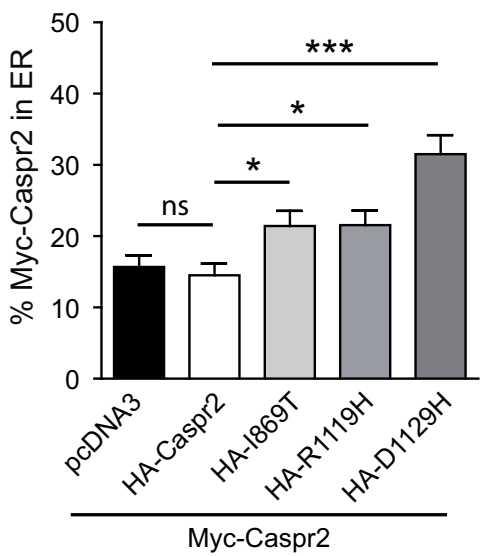

Figure 3. The variants I869T, R1119H and D1129H oligomerize with WT Caspr2 and have a dominant-negative effect on its subcellular localization in transfected COS-7 cells. (A) Schematic domain organization of HA-Caspr2 and positions of the variants. (B) Percentage of co-localization with the ER marker of WT or variant HA-Caspr2 expressed in transfected COS-7 cells. (C) Immunoprecipitation (IP) from lysates of transfected COS-7 cells co-expressing Myc-Caspr2 and WT or variant HA-Caspr2 with HA antibodies and revealed by immunoblotting (IB) with Myc or HA antibodies. Crude protein extracts (Lysates) were immunoblotted to verify protein expression. The proportion of Myc-Caspr2 co-immunoprecipitated with the variants is higher than that pulled down with HA-Caspr2. Molecular mass markers positions are shown in $\mathrm{kDa}$ on the left of the panels. (D, E) Percentage of co-localization with the ER marker of WT or variant HA-Caspr2 co-expressed with Myc-Caspr2 (D), and of Myc-Caspr2 expressed alone (pcDNA3) or co-expressed with WT or variant HA-Caspr2 (E). (B, D, E) Data are means + SEM of two experiments, 10 cells/condition/experiment. Statistical analyses: (B, D) Mann-Whitney test to compare each variant to HA-Caspr2; (E) Kruskal-Wallis one-way ANOVA test to compare pcDNA3, HA-Caspr2 and each variant condition, Dunn's Multiple Comparison post-test, ${ }^{*} \mathrm{P}<0.05,{ }^{* *} \mathrm{P}<0.01,{ }^{* * *} \mathrm{P}<0.0001,{ }^{* * * *} \mathrm{P}<0.0001$; ns, not significant.

Caspr2-Fc chimera bound specifically the surface of TAG-1expressing cells after incubation at $37^{\circ} \mathrm{C}$ for $30 \mathrm{~min}$ (Fig. 5B), without any detectable endocytosis at this time point (Supplementary Material, Fig. S4). Similar binding was observed for the variants N407S, N418D, Y716C and R906H (Fig. 5C-F). In contrast, binding was dramatically decreased for the variant I869T (Fig. 5G), thus supporting the fact that its phenotype in axon growth may be caused by cell adhesion defects. Interestingly, binding was also strongly impaired for the variant G731S (Fig. 5H). Since this variant is localized in the fibrinogen domain, we further tested its ability to form oligomers with WT Caspr2 in COS-7 cells. In contrast to HA-I869T (Fig. 3C), the proportion of the immature form of Myc-Caspr2 co-immunoprecipitated with HA-G731S was similar to that pulled down with HA-Caspr2 (Fig. 6A). In addition, immunostaining confirmed that HA-G731S was not significantly retained in the ER and did not induce ER retention of Myc-Caspr2 (Fig. 6B and C; Supplementary Material, Figs S2E and S3E). We thus evaluated its impact on axonal growth of WT, HET and KO cortical neurons. Consistent with the observations in COS-7 cells, HA-G731S was expressed at the surface of the neurons similarly to WT Caspr2 (Fig. 7A and B). Measurements showed that HAG731S did not rescue axon growth defects of HET and KO neurons (Fig. 7D). In addition, whatever the genotype of the neurons, axon growth was not significantly different from that of neurons 

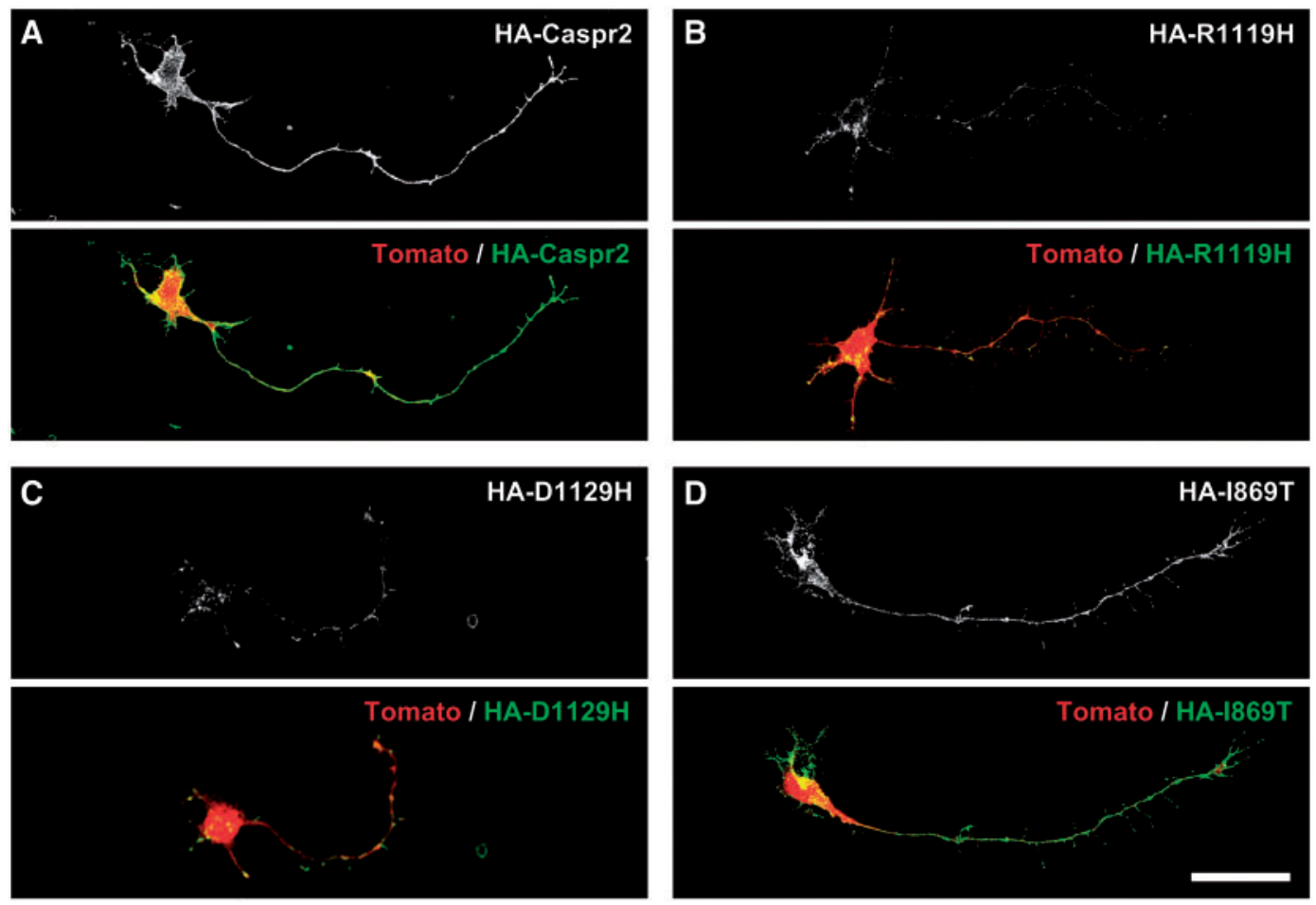

$\mathbf{E}$

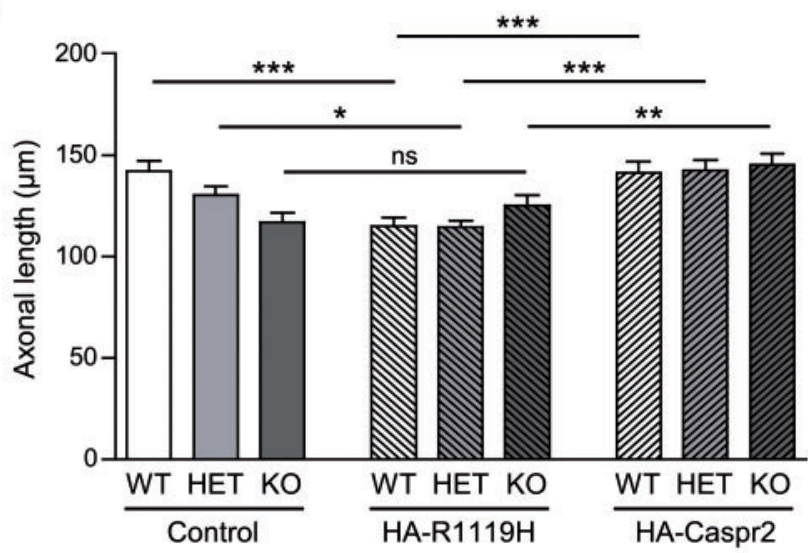

$\mathbf{F}$

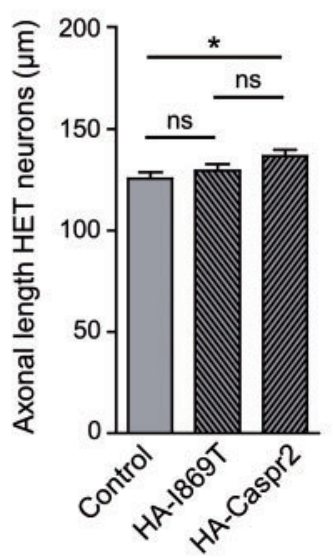

Figure 4. The variant R1119H has a dominant negative effect on axon growth while the variant I869T does not fully rescue axon growth defects of HET neurons. (A-D) Representative images of cell surface immunostaining of electroporated HET neurons co-expressing Tomato and HA-Caspr2 (A) or the variants HA-R1119H (B), HA-D1129H (C) and HA-I869T (D). HA-I869T is expressed at the plasma membrane as well as HA-Caspr2 whereas HA-R1119H and HA-D1129H are poorly detectable at the plasma membrane. E. Quantification of axon length at DIV3 of WT, HET and KO neurons electroporated with the control vector or vectors expressing HA-Caspr2 or the variant HA-R1119H. F. Quantification of axon length at DIV3 of HET neurons electroporated with the control vector or vectors expressing HA-Caspr2 or the variant HA-I869T. (E, F) Data are means + SEM. (E) $n=4-6$ embryos/genotype, $n=42-46$ neurons/embryo. (F) $n=6$ embryos/genotype, $n=31-36$ neurons/embryo. Statistical analyses: Kruskal-Wallis one-way ANOVA test, (E) $P<0.001$, (F) $P<0.05$; Dunn's Multiple Comparison post-test, ${ }^{*} P<0.05$, ${ }^{* *} P<0.01,{ }^{* * *} P<0.001$; ns, not significant. (A-D) Scale bar, $25 \mu \mathrm{m}$.

electroporated with the control vector. This indicates that, similarly to I869T, the variant G731S presents a loss-of-function in axonal outgrowth, likely because of cell adhesion defects although with probably minor structural changes as compared to I869T.

The cell-membrane variant HA-N407S has a dominant-negative effect in axonal outgrowth

Our approach using Caspr2-Fc chimera was designed to screen for protein structural changes in variants expressed at the plasma membrane but faced limits to reveal subtle physiologically relevant cell-adhesion defects or any other impairments. Thus, in a last step, we decided to evaluate directly whether one of the four variants which interacted in trans with TAG-1 (N407S, N418D, Y716C and R906H) may affect cortical neuron axon growth. We arbitrarily choose the variant N407S, which appeared to present minor trafficking defects (Supplementary Material, Fig. S1A-C). COS-7 cell studies confirmed that HAN407S was not significantly retained in the ER and did not induce ER retention of Myc-Caspr2 (Fig. $6 \mathrm{D}$ and E; Supplementary Material, Figs S2F and S3F). In addition, the 
A

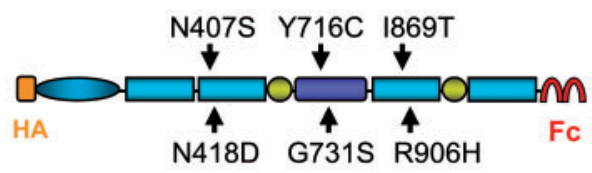

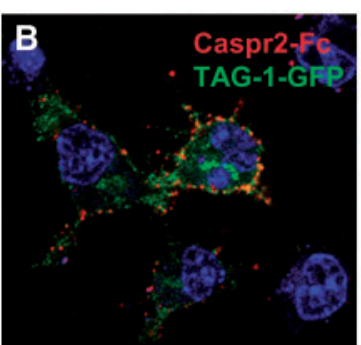
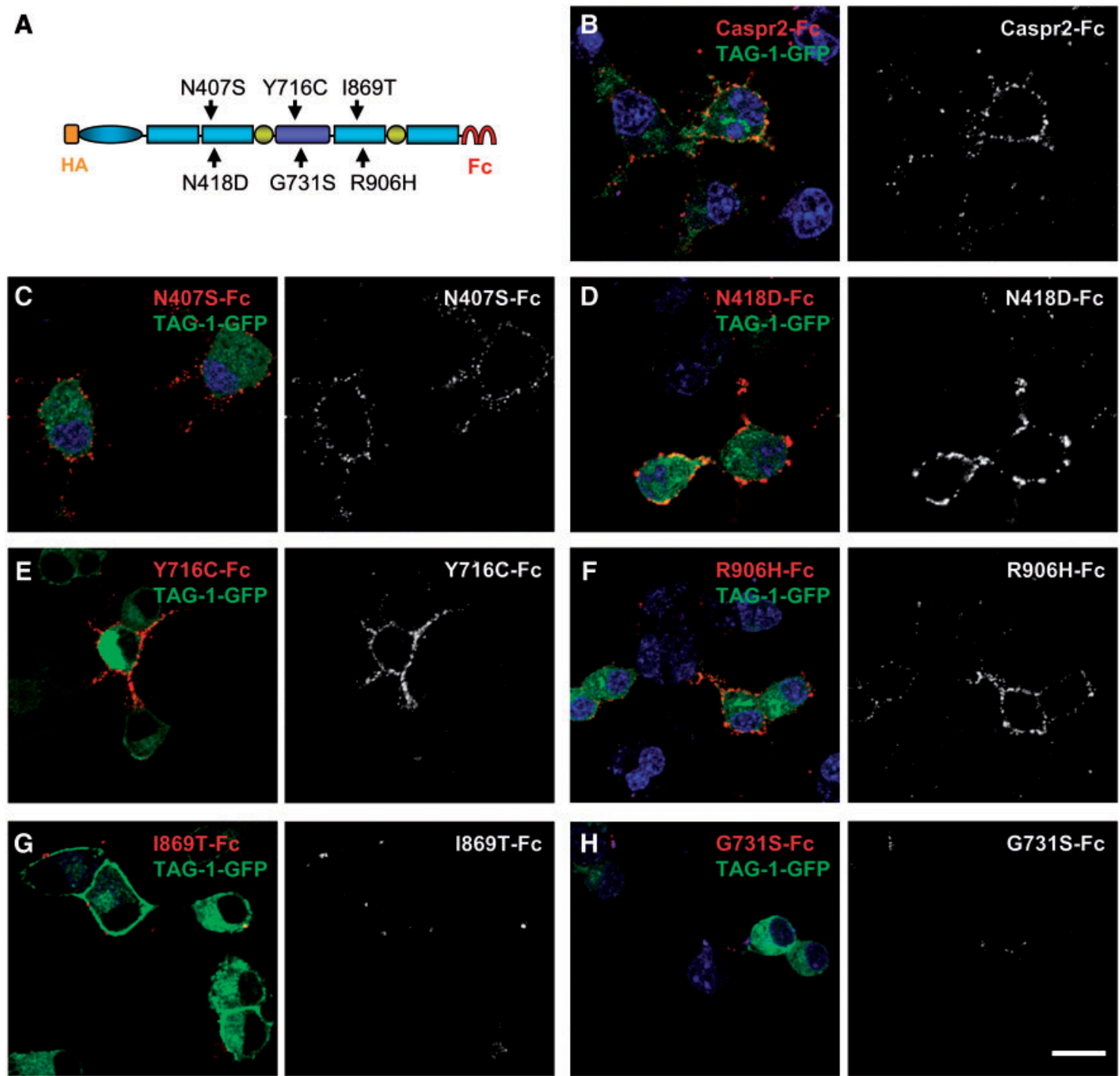
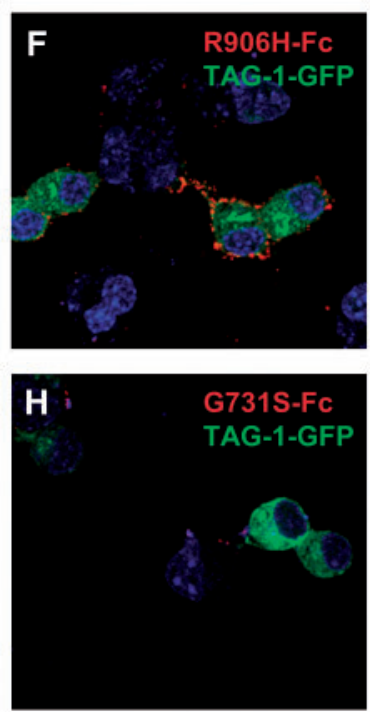
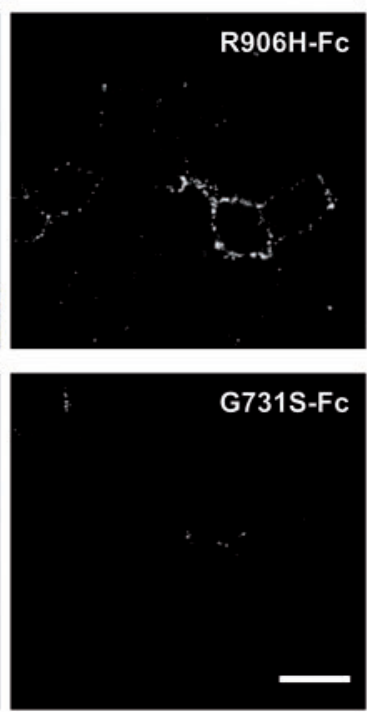

Figure 5. The variant fusion proteins G731S-FC and I869T-Fc do not bind in trans GFP-tagged TAG-1 expressed in N2a cells. (A) Schematic domain organization of Caspr2-Fc and position of the variant missense mutations. (B-H) Binding of Caspr2-Fc (B) or the variants N407S-Fc (C), N418D-FC (D), Y716C-FC (E), R906H-Fc (F), I869TFc (G) and G731S-Fc (H) (red) on N2a cells expressing GFP-TAG-1 (green). Single optical sections of confocal images. Blue, Hoechst staining, nuclei. (B, H) Scale bar, $20 \mu \mathrm{m}$.

proportion of the immature form of Myc-Caspr2 co-immunoprecipitated with this variant was similar to that pulled down with HA-Caspr2 (Fig. 6A). Cell surface immunostaining showed that HA-N407S was expressed at the surface of HET neurons similarly to WT Caspr2 (Fig. 7A and C). However and unexpectedly, axon growth of HA-N407S-expressing HET neurons was significantly decreased as compared to neurons electroporated with the control vector or expressing exogenous WT Caspr2 (Fig. 7E), suggesting that this variant has a dominant-negative effect in axonal outgrowth, in spite of its normal surface expression and probably mild or no structural changes.

\section{Discussion}

Previous studies indicated that Caspr2 may play several roles during post-natal development of cortical neurons and contribute to normal neuronal network assembly and activity (14-19). In this study, we demonstrate a new role for Caspr2, earlier during embryonic cortical neuron differentiation, showing that
Caspr2 regulates axon growth. Importantly, we find that a haplo-insufficiency reducing the expression of Caspr2 to about half is sufficient to elicit axon growth defects. This offered a reliable assay to identify the effects of ASD-related heterozygous CNTNAP2 missense variants. These mutants displayed either a dominant-negative effect or a loss-of-function during axon growth in a Cntnap2 heterozygous genetic background. Thus, our results provide evidence that CNTNAP2 variants could be clinically relevant in the pathogenicity in ASD.

During embryonic development, axon growth and guidance are early events essential for establishing the basal brain connectivity. They control the development of projections between the cortex and other brain regions, as well as cortico-cortical projections forming the corpus callosum, which is a major myelinated interhemispheric tract allowing bilateral integration of lateralized sensory inputs and regulation of higher-order cognitive, social and emotional processing (27). The cell-autonomous function of Caspr2 in axon growth that we describe was observed in cortical neurons from embryos at E14.5, a stage of active axon 
A

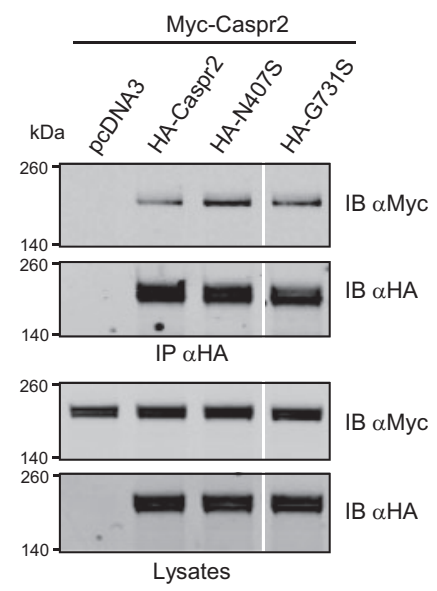

B

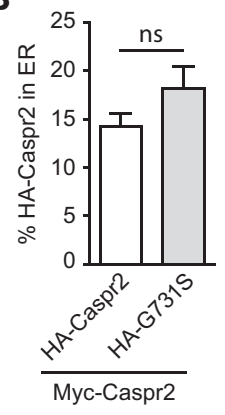

D

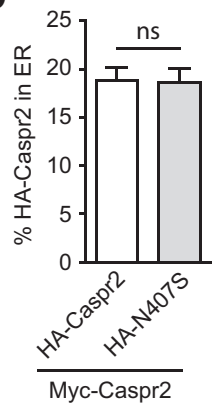

C

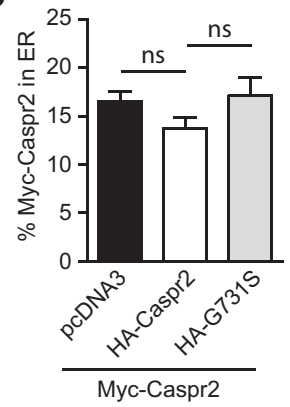

E

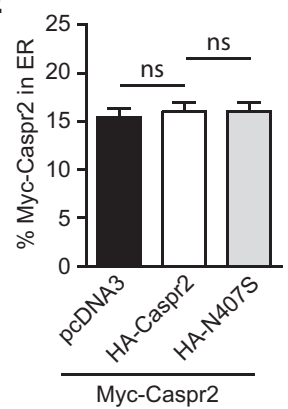

Figure 6. The variants G731S and N407S oligomerize with WT Caspr2 but do not induce ER retention of Myc-Caspr2 in transfected COS7 cells. (A) Immunoprecipitation (IP) from lysates of transfected COS-7 cells co-expressing Myc-Caspr2 and WT or the variants HA-N407S and HA-G731S with HA antibodies and revealed by immunoblotting (IB) with Myc or HA antibodies. Crude protein extracts (Lysates) were immunoblotted to verify protein expression. The proportion of the immature form of Myc-Caspr2 co-immunoprecipitated with the variants is similar to that pulled down with HA-Caspr2. Molecular mass markers positions are shown in kDa on the left of the panels. (B, C) Percentage of co-localization with the ER marker of HA-Caspr2 or HA-G731S co-expressed with Myc-Caspr2 (B), and of Myc-Caspr2 expressed alone (pcDNA3) or co-expressed with HA-Caspr2 or HA-G731S (C). (D, E) Percentage of co-localization with the ER marker of HA-Caspr2 or HA-N407S co-expressed with MycCaspr2 (D), and of Myc-Caspr2 expressed alone (pcDNA3) or co-expressed with HA-Caspr2 or HA-N407S (E). (B-E) Data are means + SEM of two experiments, 10 cells/ condition/experiment. Statistical analyses: (B, D) Mann-Whitney test to compare each variant to HA-Caspr2; (C, E) Kruskal-Wallis one-way ANOVA test to compare pcDNA3, HA-Caspr2 and variant conditions, Dunn's Multiple Comparison post-test, ns, not significant.

growth in the mouse, strongly supporting the hypothesis that Caspr2 could contribute to the normal development and patterning of cortical projections in vivo. Consistent with this hypothesis, Scott et al. recently showed that 3-week old Cntnap2 ${ }^{-/-}$mice do not present obvious morphological abnormalities of the corpus callosum, but a decrease in the density of myelinated axons in the superficial layers of the cortex, which is compensated at adulthood (14). This phenotype, reflecting a delay in myelination, may be a direct consequence of a delay in the development of the axons, which not only support myelination but also stimulate the proliferation of oligodendrocyte precursor cells through their electrical activity (28). During development, axon growth delay may influence networks dynamics of cortical neurons and perturb long-distance functional connectivity, as previously described in adult ASD patients in whom long-range corticocortical functional and structural connectivity appears to be weaker than in controls $(3,4)$. It might also contribute to transient defects in the corpus callosum observed in young toddlers later diagnosed with ASD $(29,30)$. These observations argue that CNTNAP2-associated axon growth anomalies may contribute to the manifestations of ASD.

Axon elongation is mainly triggered by the navigation of growth cones, which sense a variety of surrounding environmental signals. It is controlled by a combination of complex mechanisms working in concert, including integration of extracellular signals into intracellular second messenger networks and downstream remodelling of cytoskeletal components driving growth cone mobility (31). Our data showing that Cntnap $2^{+-}$cortical neurons exhibit an intermediate axon growth phenotype between WT and Cntnap $2^{-1-}$ neurons indicate that Caspr2 may act as a modulator in these processes. Adhesive contacts during axon growth are mainly triggered by cadherins, integrins and members of the immunoglobulin superfamily (IgSF-CAMs) (32-35). Caspr2 is known to interact in mature myelinated axons with the IgSF-CAM CNTN2/ Contactin2/TAG-1 through its ectodomain and with protein 4.1B that associates with the spectrin/actin cytoskeleton through its cytoplasmic tail $(11,12,36)$. It is therefore possible that Caspr2 modulates axon growth by interacting with IgSF-CAMs and regulating cell adhesion and cytoskeleton remodelling at growth cones and along the axon. Remarkably, TAG-1 promotes neurite outgrowth but only when presented as substrates or as soluble molecules (37), suggesting that it might not cooperate in cis with Caspr2 in axon growth. Beside IgSF-CAMs, we showed that $\beta 1$ integrin is able to interact with another member of the Caspr family, Caspr1/paranodin (38), raising the intriguing possibility that Caspr2 could also regulate integrin-based adhesions, cytoskeleton dynamics and signalling upon binding to molecules of the extracellular matrix (32). Other studies indicate that axon growth and guidance could be modulated by voltage-gated potassium channel activity $(39,40)$. Since Caspr2 is required for Kv1 clustering in myelinated axons $(11,14)$, it could be possible that, in addition or alternatively, it regulates Kv1 surface expression, localization and/or activity during axon elongation. 

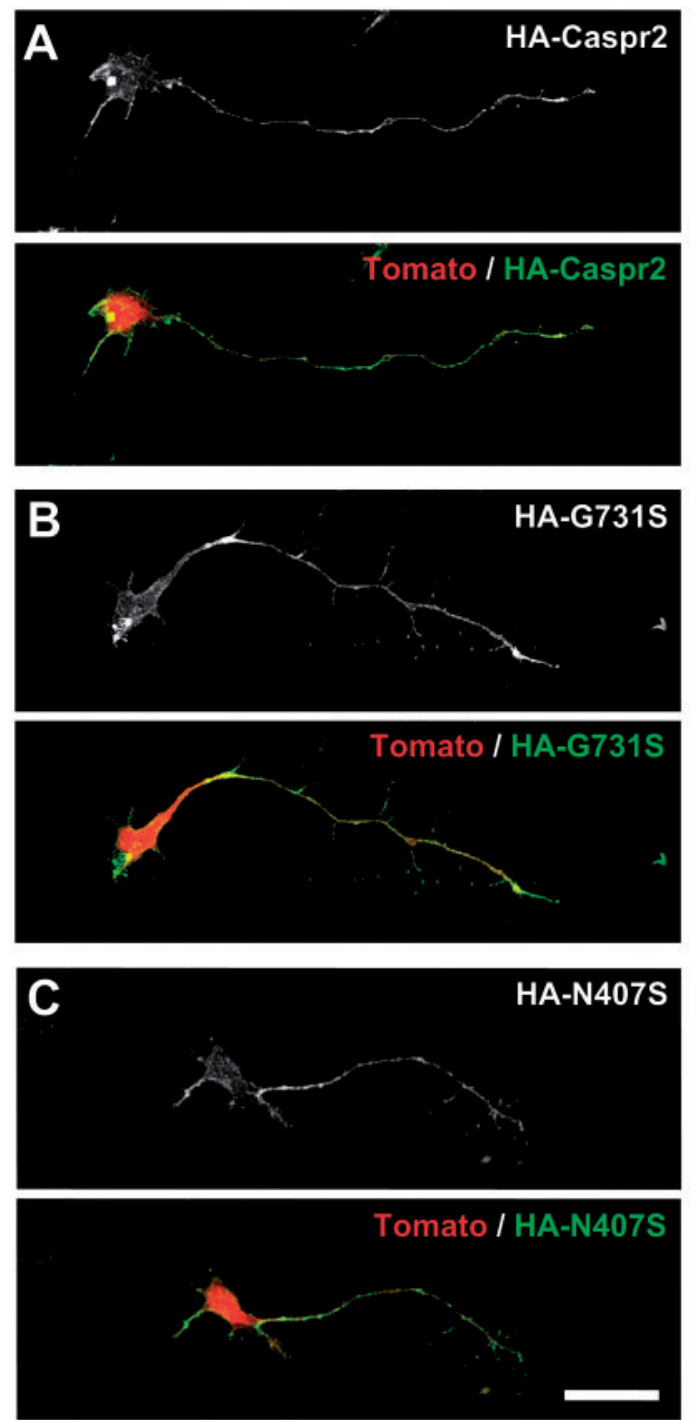

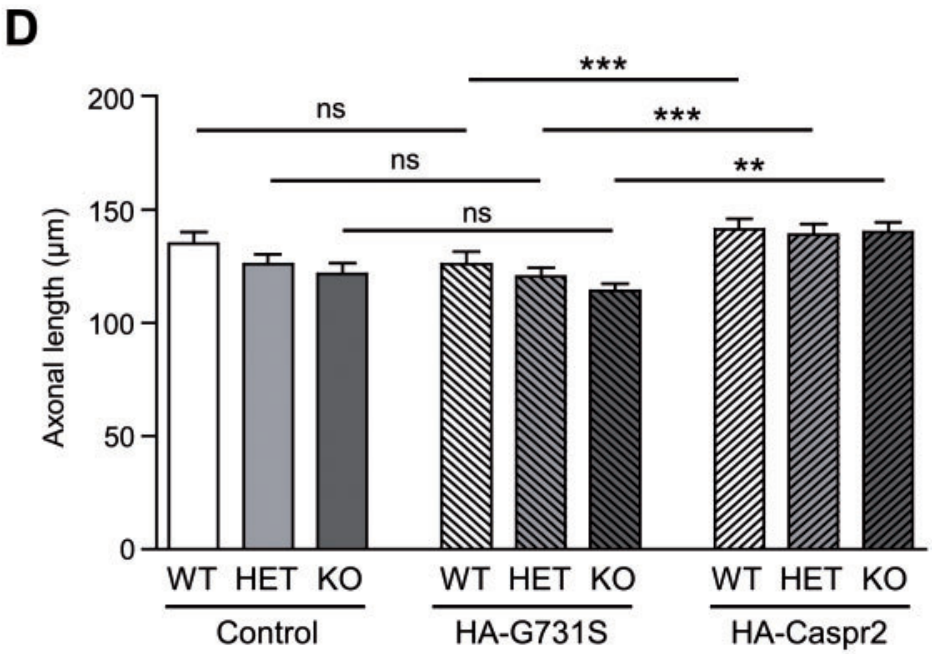

E

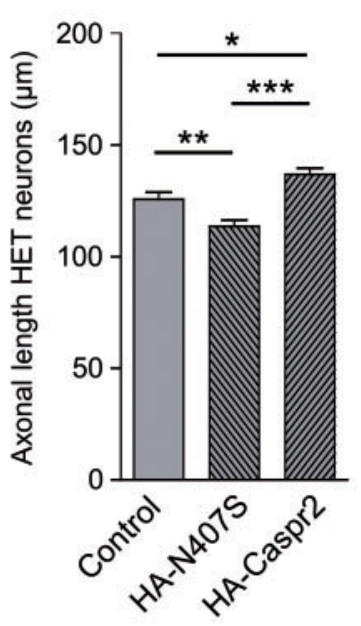

Figure 7. The variant G731S is defective in axon growth while the variant N407S has a dominant-negative effect on axon growth. (A-C) Representative images of cell surface immunostaining of HET electroporated neurons co-expressing Tomato and HA-Caspr2 (A) or the variants HA-G731S (B) and HA-N407S (C). HA-G731S and HAN407S are expressed at the plasma membrane as well as HA-Caspr2. (D) Quantification of axon lengths at DIV3 of WT, HET and KO neurons electroporated with the control vector or vectors expressing HA-Caspr2 or the variant HA-G731S. (E) Quantification of axon length at DIV3 of HET neurons electroporated with the control vector or vectors expressing HA-Caspr2 or the variant HA-N407S. (D, E) Data are means + SEM. (D) $n=3-5$ embryos/genotype, $n=35-40$ neurons/embryo. (E) $n=6$ embryos/ genotype, $n=31-36$ neurons/embryo. Statistical analyses: Kruskal-Wallis one-way ANOVA test, (D) $P<0.001$, (E) $P<0.001$; Dunn's Multiple Comparison post-test, ${ }^{*} \mathrm{P}<0.05,{ }^{* *} \mathrm{P}<0.01,{ }^{* * *} \mathrm{P}<0.001$; ns, not significant. (A-C) Scale bar, $25 \mu \mathrm{m}$.

While genetic data question the clinical significance of CNTNAP2 variants in ASD, our study using axon growth as readout demonstrates for the first time that some mutations have functional consequences in a genetic background relevant for CNTNAP2 heterozygosity in ASD. The variants G731S and I869T, which likely present structural changes preventing their interactions with cell adhesion partners, do not rescue the heterozygous axon growth phenotype, indicating that phenotypes related to CNTNAP2 heterozygosity may exist in human. The misfolded ER-retained variant $\mathrm{R} 1119 \mathrm{H}$ plays a dominantnegative effect in axon growth, arguing that phenotypes mimicking homozygous CNTNAP2 null mutation may also exist in human. Falliveli et al. previously demonstrated that variants of this category could associate with chaperones in the ER, leading to stimulation of the unfolded protein response and subsequent proteasomal degradation (25). We found that they are able to oligomerize with WT Caspr2 and induce its ER retention, thus leading to a lack or decrease of functional protein at the plasma membrane. We identified a third type of variants, N407S, which is well-localized at the membrane but also appears to play a dominant-negative effect on axon growth. This variant is not expected to exert a direct effect on WT Caspr2 since it does not interact with the plasma membrane form (Fig. 6A), indicating that it impairs WT Caspr2 functions through a mechanism that remains to be identified. Altogether these data show that CNTNAP2 variants can impact axon growth in a heterozygous background through at least three different mechanisms, and may therefore be responsible for a variety of phenotypes in human. Of note, experiments in COS-7 cells further suggest that the variants likely display a gradient of intracellular retention rather than being either strictly retained in the ER or strictly addressed at the plasma membrane, raising the possibility that 
all CNTNAP2 variants identified so far could induce a continuum of phenotypes rather than discrete phenotypes strictly mimicking CNTNAP2 heterozygous or homozygous null mutation.

Our observations further predict that CNTNAP2 variants may lead to a high heterogeneity of phenotypes at the scale of the whole brain. In line with the dose-dependent function of Caspr2 in axon growth, Cntnap2 ${ }^{+/-}$fast-spiking $\mathrm{PV}^{+}$cortical interneurons exhibit an intermediate electrophysiological phenotype between WT and Cntnap2 ${ }^{-1-}$ interneurons (15), suggesting that several functions of Caspr2 may be dose-sensitive. In contrast, the maturation of $\mathrm{PV}^{+}$GABAergic cortical interneurons is only altered in Cntnap $2^{-/-}$mice, indicating that other functions may not be affected by a partial deficit in Caspr2 (15). This implies that Caspr2 functions may be differentially affected in each individual variant, depending both on their sensitivity to Caspr2 level and the direct impact of the variants on the structure, trafficking and/or interactions of the protein. Consistently, the variants N407S and G731S do not impair interneuron maturation in a Cntnap $2^{+/-}$genetic background although they demonstrate a decrease/loss-of-function in a Cntnap $2^{-1-}$ genetic background (15).

The potential impact of CNTNAP2 variants was until now evaluated by bioinformatics analyses using SIFT and Polyphen2 software, which predict whether an amino acid substitution may affect protein structure. Consistent with our experimental results, these programs predicted that the variants R1119H and G731S may be 'damaging' and/or 'probably damaging', respectively (20). In contrast, the variants N407S and I869T were predicted to be 'tolerated' and 'benign' (20) and therefore not expected to induce major functional impairments such as we observed. This discrepancy may find an explanation in the fact that the extracellular domain of Caspr2 presents a very compact subdomain organization, probably flexible upon protein partner binding, and therefore complex structure-function relationships $(23,24)$. The variant I869T, which is localized in a laminin G domain, may not induce a predictable structural disorganization of this domain but a disorganization of the overall structure of the protein by disrupting inter-domain interactions. The variant N407S, which seems to have a mild effect on the structure, may be in an exposed region essential for partner interactions other than CNTN2/Contactin2/TAG-1. Without available high resolution structural information for Caspr2, these observations raise the important question of the limits of bioinformatics predictions, especially for the variants which have been predicted to be non-deleterious and which represent a large proportion of all CNTNAP2 variants identified so far. Furthermore, they strongly support the hypothesis that more variants than originally predicted may be functionally deleterious, and thus highlight the possibility that CNTNAP2 variants may define an overall endophenotype shaping a risk for ASD.

Recent genetic studies did not find evidence for statistical differences for CNTNAP2 variants between ASD and control subjects (20). Similarly to the variants identified in ASD patients, the variants found in controls are distributed over the entire extracellular domain of Caspr2, and some of them are predicted to be 'deleterious' and/or 'probably damaging' by SIFT and Polyphen-2 software, respectively. However, none of the variants we examined here, including the variant I869T identified in several ASD families $(20,21)$, were found in controls. It is therefore possible that only some point mutations in CNTNAP2 have biological consequences and clinical relevance. Alternatively, it is also possible that the variants found in controls have biological consequences similar to that of ASD variants but in genetic backgrounds which do not favour to the manifestations the pathology, as it is likely the case for unaffected parents of ASD patients. Systematic investigation of many CNTNAP2 variants in in vitro assays such as those we have used will help clarifying this issue.

In conclusion, our data provide a proof of principle that CNTNAP2 heterozygous variants may contribute to the pathophysiology of ASD. They further underline the importance to pursue the characterization of the functional and behavioural consequences of missense variants in a heterozygote background in vivo as well as to evaluate their interaction with additional genetic and/or environmental risk factors for the development of ASD-related phenotypes. Moreover, considering the large number of variants identified in ASD patients and in the control population, they point at the necessity to resolve the structure of Caspr2 to improve the predictions of the potential impact of all variants.

\section{Materials and Methods}

\section{Antibodies}

The rabbit antibody directed against the intracellular domain of Caspr2 (residues 1284-1331) and purified anti-Caspr2 IgGs from a patient affected by an autoimmune limbic encephalitis were previously described $(26,36)$. Commercial primary antibodies were from the following sources: Myc, rabbit \#2272 or mouse clone 9B11 \#2276, Cell Signalling; HA, rat clone 3F10 \#11 867423 001, Roche; KDEL, mouse \#SPA-827, Stressgen; Class III $\beta 3$ tubulin, mouse clone TUJ1 \#MMS-435P, Covance; Actin, mouse clone C4 \#MAB1501, Merck-Millipore. Secondary antibodies for immunohistochemistry and phalloidin (Alexa Fluor 488, 546, 568 or 633 conjugated) were from Thermo Fisher scientific. IRDye ${ }^{\mathrm{TM}} 800 \mathrm{CW}$ - and IRDye ${ }^{\mathrm{TM}} 700 \mathrm{CW}$-conjugated secondary antibodies for immunoblotting and IRDye ${ }^{\mathrm{TM}} 800 \mathrm{CW}$-conjugated streptavidin were from Rockland Immunochemicals.

\section{Plasmid constructs}

The pCDNA-HA-Caspr2 and pCDNA-Myc-Caspr2 constructs encode human Caspr2 (Accession number NM_014141, KIAA0868) with the HA epitope and the Myc epitope, respectively, inserted downstream of the signal peptide between the residues Trp26 and Thr27, in PCDNA3 and PCDNA5/FRT vectors, respectively. The Caspr2-Fc construct encodes the human Caspr2 extracellular domain (amino acids 1-1242) inserted upstream the human Fc sequence in the pIg-plus vector (26). The pCDNA-HA-Caspr2 deleted constructs Caspr2 $\Delta 1$ ( $\Delta 32-361)$, Caspr2 $\Delta 2$ ( $\Delta 362-600)$, Caspr2 $\Delta 3(\Delta 600-950)$ and Caspr $2 \Delta 4$ ( $\Delta 955-1169)$ were previously described (26). Mutated pCDNAHA-Caspr2 and Caspr2-Fc constructs expressing Caspr2 variant proteins were generated by site-directed mutagenesis using specific oligonucleotides (Supplementary Material, Table S1). Constructs for expression of WT and variant Caspr2 in cortical neurons were generated by subcloning the cDNAs coding for the HA-tagged proteins in the vector PCAGGS-IRES-Tomato (generous gift from Sophie Lebrand) upstream the IRES sequence. The TAG-1-GFP vector encodes human TAG-1 (Accession number X67734) with the GFP tag inserted downstream the signal peptide (26).

\section{Animals}

Cntnap2 mutant mice, previously described (11), were obtained from the Jackson Laboratory and maintained in a C57BL/6J 
background. They were group housed with ad libitum access to food and water and a $12 \mathrm{~h}-12 \mathrm{~h}$ light-dark cycle (light phase onset at 7 a.m.). For staging of embryos, the day of vaginal plug was considered E0.5. All the experiments were approved by the French Agriculture and Forestry Ministry (C75-05-22).

\section{Primary cultures of dissociated cortical neurons}

For cortical neuron cultures, the cortex from mouse E14.5 embryos was dissected in ice-cold $0.02 \mathrm{M} \mathrm{HEPES}$ in $\mathrm{Ca}^{2+} / \mathrm{Mg}^{2+}$ free HBSS (Sigma), and mechanically dissociated after trypsin $(2.5 \mathrm{mg} / \mathrm{ml})$ incubation. For axonal growth analysis, dissociated cells were plated on $14 \mathrm{~mm}$-diameter coverslips $\left(0.3 \times 10^{5}\right.$ cells/ coverslip) coated with poly-L-lysine $(0.05 \mathrm{mg} / \mathrm{ml}$, Sigma) and natural mouse laminin $(0.01 \mathrm{mg} / \mathrm{ml}$, Invitrogen), and cultured in Neurobasal medium supplemented with B27 serum-free supplement $\left(20 \mu \mathrm{l} / \mathrm{ml}\right.$, Gibco $\left.^{\circledR}\right)$, Glutamax $(10 \mu \mathrm{l} / \mathrm{ml}$, Invitrogen), penicillin $(100$ units $/ \mathrm{ml})$ and streptomycin $(100 \mu \mathrm{g} / \mathrm{ml})$ at $37^{\circ} \mathrm{C}$ in presence of $5 \% \mathrm{CO}_{2}$. To express WT Caspr2 and variants, pCAGGS-HACaspr2-IRES-Tomato constructs were electroporated in neurons $\left(1.25 \times 10^{5}\right.$ cells/coverslip) before plating using the Neon Transfection System (Invitrogen) according to the manufacturer's protocol (two 20-ms pulses, $1350 \mathrm{~V}$ ). For Cntnap2 and Caspr2 expression analyses, dissociated neurons were plated in in 6-well plates coated with poly-L-lysine and laminin $\left(5 \times 10^{5}\right.$ cells/well).

\section{Cell line cultures, transfections, tunicamycin treatment and Caspr2-fc binding}

For co-immunoprecipitation experiments and sub-cellular localization analyses, COS-7 grown in DMEM containing $10 \%$ foetal calf serum were transiently transfected using Polyethylenimine (PEI), using $10 \mu \mathrm{g}$ of plasmid/30 $\mu \mathrm{g} \mathrm{PEI} / 2 \times 10^{6}$ cells/100 mm-diameter dish and $1 \mu \mathrm{g}$ of plasmid/3 $\mu \mathrm{g} \mathrm{PEI} / 5 \times 10^{4}$ cells/20 mm-diameter coverslip, respectively. After transfection, cells were grown for $24 \mathrm{~h}$ before processing or before treatment with $2 \mu \mathrm{g} / \mathrm{ml}$ tunicamycin (or DMSO as control) during an additional period of $18 \mathrm{~h}$. Caspr2-Fc fusion protein binding experiments were performed as previously described (26). Briefly, WT and variant Caspr2-Fc fusion proteins were produced in the supernatant of transfected HEK-293 cells, affinity purified using Protein-A Sepharose, pre-clustered with Alexa Fluor conjugated anti-human FC IgGs and incubated with N2a cells expressing GFP-tagged TAG-1 for $30 \mathrm{~min}$ at $37^{\circ} \mathrm{C}$, before cell fixation with paraformaldehyde. Using these conditions, Caspr2-Fc was only detected at the surface of TAG-1-expressing cells without any detectable endocytosis.

\section{Biochemical experiments}

To analyze endogenous expression of Caspr2 or expression of WT and variant HA-Caspr2 in transfected COS-7, brains, cortical neurons and cells were lysed in RIPA buffer (50 mm Tris, pH 8.0, $150 \mathrm{~mm} \mathrm{NaCl}, 10 \mu \mathrm{l} / \mathrm{ml} \mathrm{NP}-40,5 \mu \mathrm{g} / \mathrm{ml}$ sodium deoxycholate, $1 \mu \mathrm{g} / \mathrm{ml}$ SDS) containing Complete protease inhibitors (Roche). Equal amounts of proteins were load on NuPAGE 8-12\% Bis-Tris gels (Thermo Fisher scientific) and transferred to $0.45 \mu \mathrm{m}$ Nitrocellulose membrane in $25 \mathrm{~mm}$ Tris-HCl, pH 7.4, $192 \mathrm{~mm}$ glycine and $200 \mathrm{ml} / \mathrm{l}$ ethanol. Membranes were blocked with 50 g/l non-fat dry milk in Tris-buffered saline (TBS; $0.25 \mathrm{M}$ Tris/0.5 $\mathrm{M} \mathrm{NaCl}, \mathrm{pH}$ 7.5)-Tween $1 \mathrm{ml} / \mathrm{l}$ (TBST) for $1 \mathrm{~h}$ at room temperature, incubated with primary antibodies in the same buffer for
$2 \mathrm{~h}$ and then $1 \mathrm{~h}$ with appropriate IRDye-conjugated secondary antibodies, and imaged and quantified using Odyssey Imaging System (LI-COR Biosciences). For endoglycosidase digestions, transfected cells were lysed in a buffer containing $50 \mathrm{~mm}$ Tris, $\mathrm{pH}$ 7.4, $30 \mathrm{mM} \mathrm{NaCl}, 1 \mathrm{mM}$ EDTA, $10 \mu \mathrm{l} / \mathrm{ml}$ Triton X-100, Complete protease inhibitors (Roche) and $1 \mathrm{mM}$ phenylmethanesulfonyl fluoride (PMSF). After centrifugation, protein extracts were supplemented with SDS (final concentration $1 \mu \mathrm{g} / \mathrm{ml}$ ), $\beta$ mercaptoethanol (final concentration $10 \mu \mathrm{l} / \mathrm{ml}$ ) and PMSF (final concentration $1 \mathrm{mM}$ ), and equal amounts $(50 \mu \mathrm{l})$ were digested at $37^{\circ} \mathrm{C}$ for $3 \mathrm{~h}$ with endoglycosidase $\mathrm{H}$ (Roche; $1 \mathrm{mU}$ ) and endoglycosidase F (Roche; $43 \mathrm{mU}$ ), respectively, before loading on gels. Co-immunoprecipitations were performed essentially as previously described (12). The extraction buffer contained 85 mM Tris, pH 7.5, $30 \mathrm{mM} \mathrm{NaCl}, 1 \mathrm{mM}$ EDTA, 120 mM glucose, 10 $\mu \mathrm{l} / \mathrm{ml}$ Triton X-100 and Complete protease inhibitors (Roche). The washing buffer contained $50 \mathrm{mM}$ Tris, pH 7.5, $150 \mathrm{mM} \mathrm{NaCl}$, $5 \mu \mathrm{l} / \mathrm{ml}$ Triton X-100 and Complete protease inhibitors. For cell surface biotinylation experiments, transfected cells were washed three times for 10 min with PBS at $4^{\circ} \mathrm{C}$ and then biotinylated with $0.5 \mathrm{mg} / \mathrm{ml}$ sulfo-NHS-LC biotin (Pierce) in $10 \mathrm{mM}$ triethanolamine, $\mathrm{pH} 9,140 \mathrm{mM} \mathrm{NaCl}$ for $20 \mathrm{~min}$ at $4^{\circ} \mathrm{C}$. After two washes in PBS at $4^{\circ} \mathrm{C}$, cells were lysed and immunoprecipitation was carried out as described above. After migration and transfert, membranes were blotted with IRDye ${ }^{\mathrm{TM}} 800 \mathrm{CW}$ conjugated streptavidin to detect biotinylated Caspr2.

\section{Cntnap2 mRNA expression analysis}

For mRNA expression analysis, total mRNA was extracted from brains and cortical neurons in culture using the TRIzol ${ }^{\circledR}$ Reagent (Life Technologies) following the manufacturer's recommendations. Reverse transcription (RT) was performed with the SuperScript II reverse transcriptase (Invitrogen, \#18064-022) and random primers. Primers for Caspr2 cDNA amplification (PCR) were designed to amplify a 476-bp DNA fragment overlapping the 5 ' non-coding region: primer sens (5'-CAGCGAGCTTTTGGA GTACCACTG), nucleotides 191 to 214 of mouse cDNA (accession number NM_001004357); primer anti-sens (5'-GACCACGCTGT CAGAATTGACGTT), nucleotides 644 to 667 . For expression analysis of the housekeeping gene peptidylpropyl isomerase $B$ (Cyclophilin B, Cyp), RT/PCR was performed using the primers ( $5^{\prime}$ CCATCGTGTCATCAAGGACTT) and (5'-TTGCCATCCAGCCAGGAG GTC). The expected size of the amplified DNA was $215 \mathrm{bp}$.

\section{Immunofluorescence staining}

For transfected COS-7 cell staining, cells were fixed with paraformaldehyde (PFA, $40 \mathrm{mg} / \mathrm{ml}$ ) in PBS for $30 \mathrm{~min}$ at room temperature (RT) and permeabilized in PBS-Triton X-100 $2 \mu \mathrm{l} / \mathrm{ml}$ for $5 \mathrm{~min}$ at RT. Coverslips were incubated in a saturation solution (50 $\mathrm{mg} / \mathrm{ml}$ bovine serum albumin in PBS), and with primary antibodies and Alexa Fluor conjugated-secondary antibodies (diluted in the saturation solution) at RT for 2 and $1 \mathrm{~h}$, respectively.

To quantify axonal growth in non-electroporated neurons, cells were fixed by adding in the medium $1 / 3$ volume of preheated PFA $(80 \mathrm{mg} / \mathrm{ml}) /$ sucrose $(200 \mathrm{mg} / \mathrm{ml})$ in PBS for $20 \mathrm{~min}$ and immunostaining was performed as previously described (41). Briefly, coverslips were incubated in a permeabilization/ saturation solution (PS; $2.5 \mu \mathrm{l} / \mathrm{ml}$ Triton X-100, $50 \mathrm{mg} / \mathrm{ml}$ bovine serum albumin, PBS) for $2 \mathrm{~h}$ at RT, with TUJ1 primary antibodies diluted in PS overnight at $4^{\circ} \mathrm{C}$, with Alexa Fluor conjugated- 
secondary antibodies diluted in PS for $2 \mathrm{~h}$ at RT, and finally with Alexa Fluor 488/546/633 conjugated-phalloidin diluted in PBS for 30 min at RT. To quantify axonal growth in neurons expressing WT or variant HA-Caspr2, coverslips were incubated with Alexa Fluor conjugated-phalloidin only.

To reveal the endogenous expression of Caspr2 and the subcellular localization of WT and variant HA-Caspr2 in cortical neurons, live neurons were incubated in the culture medium with the purified IgGs from the patient affected by an autoimmune limbic encephalitis $(20 \mu \mathrm{g} / \mathrm{ml})$ and HA antibodies, respectively, for $20 \mathrm{~min}$ at RT and fixed with $40 \mathrm{mg} / \mathrm{ml}$ PFA in PBS for $15 \mathrm{~min}$ at RT. Coverslips were incubated in a saturation solution (50 mg/ml bovine serum albumin in PBS) at RT for $30 \mathrm{~min}$ and with Alexa Fluor conjugated-secondary antibodies at RT for 60 min (endogenous expression) or $30 \mathrm{~min}$ (WT or variant HACaspr2). After three washes with PBS, neurons were fixed, permeabilized and incubated with Alexa Fluor conjugatedphalloidin as above.

\section{Image acquisition and analysis}

To monitor the subcellular localization of Myc-Caspr2 and WT or variant HA-Caspr2 in transfected COS-7 cells, images were acquired using a Leica SP5 confocal laser-scanning microscope (Leica microsystems, Z stack $1 \mu \mathrm{m}$ ) with constant parameters and colocalization analyses were performed using the software Imaris (Bitplane). The volume of the cell (Surface tool) was defined using Myc-Caspr2 or cortical actin staining to delimit the plasma membrane. The volume of the cytoplasm was isolated by subtracting the volume of the nucleus. The Mask tool was applied to the cytoplasm volume to define ER staining as Region Of Interest (ROI), which then served to determine the percentage of co-localization of Myc-Caspr2 and WT or variant HA-Caspr2 with ER using the Colocalization Tool.

For cortical neuronal morphological analyses, images were randomly acquired using an epifluorescence microscope (DM6000 or DM6002, Leica) equipped with a CCD camera and axon lengths were measured manually using Image J.

\section{Statistical analysis}

Statistical analyses were performed with GraphPad Prism 5 software. For variables that did not follow a normal distribution, statistical analyses were carried out using the Mann-Whitney rank sum test to compare 2 quantitative variables, or the Kruskal-Wallis one-way ANOVA test to compare 3 or more variables. Significant main effects were further analyzed by post hoc comparisons of means using t-tests. The significance was established at a $P$-value $<0.05$.

\section{Supplementary Material}

Supplementary Material is available at HMG online.

\section{Acknowledgements}

We are grateful to R. Boukhari, N. Roblot, Y. Bertelle, A. Rousseau, M. Savariradjane and T. Eirinopoulou (Institut du Fer à Moulin Cell and Tissue Imaging facility) for animal care and assistance with microscopes. This work was supported by Inserm, Université Pierre et Marie Curie, and grants from the Fondation Orange and the Bio-Psy laboratory of excellence. G.C., K.O. and D.P. were recipients of a doctoral fellowship from the Ministère de la Recherche et de l'Enseignement Supérieur, and a post-doctoral fellowship from the Association pour la Recherche sur la Sclérose en Plaques (ARSEP). Equipment at the IFM was also supported by DIM NeRF from Région Ile-de-France (NERF, 10016908) and by the FRC/Rotary 'Espoir en tête'. The teams of J.A.G. and L.G. are affiliated with the Paris School of Neuroscience (ENP) and the Bio-Psy laboratory of excellence.

Conflict of Interest statement. None declared.

\section{References}

1. De Rubeis, S. and Buxbaum, J.D. (2015) Recent advances in the genetics of autism spectrum disorder. Curr. Neurol. Neurosci. Rep., 15, 36.

2. Geschwind, D.H. and Levitt, P. (2007) Autism spectrum disorders: developmental disconnection syndromes. Curr. Opin. Neurobiol., 17, 103-111.

3. Vissers, M.E., Cohen, M.X. and Geurts, H.M. (2012) Brain connectivity and high functioning autism: a promising path of research that needs refined models, methodological convergence, and stronger behavioral links. Neurosci. Biobehav. Rev., 36, 604-625.

4. Rane, P., Cochran, D., Hodge, S.M., Haselgrove, C., Kennedy, D.N. and Frazier, J.A. (2015) Connectivity in autism: a review of MRI connectivity studies. Harv. Rev. Psychiatry, 23, 223-244.

5. Gilbert, J. and Man, H.Y. (2017) Fundamental elements in autism: from neurogenesis and neurite growth to synaptic plasticity. Front. Cell. Neurosci., 11, 359.

6. Van Battum, E.Y., Brignani, S. and Pasterkamp, R.J. (2015) Axon guidance proteins in neurological disorders. Lancet Neurol., 14, 532-546.

7. McFadden, K. and Minshew, N.J. (2013) Evidence for dysregulation of axonal growth and guidance in the etiology of ASD. Front. Hum. Neurosci., 7, 671.

8. Rodenas-Cuadrado, P., Ho, J. and Vernes, S.C. (2014) Shining a light on CNTNAP2: complex functions to complex disorders. Eur. J. Hum. Genet, 22, 171-178.

9. Penagarikano, O. and Geschwind, D.H. (2012) What does CNTNAP2 reveal about autism spectrum disorder?. Trends Mol. Med., 18, 156-163.

10. Poot, M. (2015) Connecting the CNTNAP2 Networks with Neurodevelopmental Disorders. Mol. Syndromol., 6, 7-22.

11. Poliak, S., Salomon, D., Elhanany, H., Sabanay, H., Kiernan, B., Pevny, L., Stewart, C.L., Xu, X., Chiu, S.Y. and Shrager, P. (2003) Juxtaparanodal clustering of Shaker-like K+ channels in myelinated axons depends on Caspr2 and TAG-1. J. Cell. Biol., 162, 1149-1160.

12. Traka, M., Goutebroze, L., Denisenko, N., Bessa, M., Nifli, A., Havaki, S., Iwakura, Y., Fukamauchi, F., Watanabe, K., Soliven, B. et al. (2003) Association of TAG-1 with Caspr2 is essential for the molecular organization of juxtaparanodal regions of myelinated fibers. J. Cell. Biol., 162, 1161-1172.

13. Poliak, S., Gollan, L., Martinez, R., Custer, A., Einheber, S., Salzer, J.L., Trimmer, J.S., Shrager, P. and Peles, E. (1999) Caspr2, a new member of the neurexin superfamily, is localized at the juxtaparanodes of myelinated axons and associates with K+ channels. Neuron, 24, 1037-1047.

14. Scott, R., Sanchez-Aguilera, A., van Elst, K., Lim, L., Dehorter, N., Bae, S.E., Bartolini, G., Peles, E., Kas, M.J.H., Bruining, H. et al. (2017) Loss of Cntnap2 causes axonal excitability deficits, developmental delay in cortical myelination, and 
abnormal stereotyped motor behavior. Cereb. Cortex. doi: 10.1093/cercor/bhx341.

15. Vogt, D., Cho, K.K.A., Shelton, S.M., Paul, A., Huang, Z.J., Sohal, V.S. and Rubenstein, J.L.R. (2017) Mouse Cntnap2 and human CNTNAP2 ASD alleles cell autonomously regulate $\mathrm{PV}+$ cortical interneurons. Cereb. Cortex, 28, 1-12.

16. Penagarikano, O., Abrahams, B.S., Herman, E.I., Winden, K.D., Gdalyahu, A., Dong, H., Sonnenblick, L.I., Gruver, R., Almajano, J., Bragin, A. et al. (2011) Absence of CNTNAP2 leads to epilepsy, neuronal migration abnormalities, and core autism-related deficits. Cell, 147, 235-246.

17. Anderson, G.R., Galfin, T., Xu, W., Aoto, J., Malenka, R.C. and Sudhof, T.C. (2012) Candidate autism gene screen identifies critical role for cell-adhesion molecule CASPR2 in dendritic arborization and spine development. Proc. Natl. Acad. Sci. U.S.A., 109, 18120-18125.

18. Varea, O., Martin-de-Saavedra, M.D., Kopeikina, K.J., Schurmann, B., Fleming, H.J., Fawcett-Patel, J.M., Bach, A., Jang, S., Peles, E., Kim, E. et al. (2015) Synaptic abnormalities and cytoplasmic glutamate receptor aggregates in contactin associated protein-like 2/Caspr2 knockout neurons. Proc. Natl. Acad. Sci. U.S.A., 112, 6176-6181.

19. Gdalyahu, A., Lazaro, M., Penagarikano, O., Golshani, P., Trachtenberg, J.T. and Gescwind, D.H. (2015) The autism related protein contactin-associated protein-like 2 (CNTNAP2) stabilizes new spines: an in vivo mouse study. PLoS One, 10, e0125633.

20. Murdoch, J.D., Gupta, A.R., Sanders, S.J., Walker, M.F., Keaney, J., Fernandez, T.V., Murtha, M.T., Anyanwu, S., Ober, G.T., Raubeson, M.J. et al. (2015) No evidence for association of autism with rare heterozygous point mutations in Contactin-Associated Protein-Like 2 (CNTNAP2), or in other contactin-associated proteins or contactins. PLoS Genet., 11, e1004852.

21. Bakkaloglu, B., O’Roak, B.J., Louvi, A., Gupta, A.R., Abelson, J.F., Morgan, T.M., Chawarska, K., Klin, A., Ercan-Sencicek, A.G., Stillman, A.A. et al. (2008) Molecular cytogenetic analysis and resequencing of contactin associated protein-like 2 in autism spectrum disorders. Am. J. Hum. Genet., 82, 165-173.

22. Poot, M. (2017) Intragenic CNTNAP2 deletions: a bridge too far?. Mol. Syndromol., 8, 118-130.

23. Rubio-Marrero, E.N., Vincelli, G., Jeffries, C.M., Shaikh, T.R., Pakos, I.S., Ranaivoson, F.M., von Daake, S., Demeler, B., De Jaco, A., Perkins, G. et al. (2016) Structural characterization of the extracellular domain of CASPR2 and insights into its association with the novel ligand contactin1. J. Biol. Chem., 291, 5788-5802.

24. Lu, Z., Reddy, M.V., Liu, J., Kalichava, A., Zhang, L., Chen, F., Wang, Y., Holthauzen, L.M., White, M.A., Seshadrinathan, S. et al. (2016) Molecular architecture of contactin-associated protein-like 2 (CNTNAP2) and its interaction with contactin 2 (CNTN2). J. Biol. Chem., 291, 24133-24147.

25. Falivelli, G., De Jaco, A., Favaloro, F.L., Kim, H., Wilson, J., Dubi, N., Ellisman, M.H., Abrahams, B.S., Taylor, P. and Comoletti, D. (2012) Inherited genetic variants in autism-related CNTNAP2 show perturbed trafficking and ATF6 activation. Hum. Mol. Genet., 21, 4761-4773.

26. Pinatel, D., Hivert, B., Boucraut, J., Saint-Martin, M., Rogemond, V., Zoupi, L., Karagogeos, D., Honnorat, J. and Faivre-Sarrailh, C. (2015) Inhibitory axons are targeted in hippocampal cell culture by anti-Caspr2 autoantibodies associated with limbic encephalitis. Front. Cell. Neurosci., 9, 265.

27. Leyva-Diaz, E. and Lopez-Bendito, G. (2013) In and out from the cortex: development of major forebrain connections. Neuroscience, 254, 26-44.

28. Barres, B.A. and Raff, M.C. (1993) Proliferation of oligodendrocyte precursor cells depends on electrical activity in axons. Nature, 361, 258-260.

29. Wolff, J.J., Gerig, G., Lewis, J.D., Soda, T., Styner, M.A., Vachet, C., Botteron, K.N., Elison, J.T., Dager, S.R., Estes, A.M. et al. (2015) Altered corpus callosum morphology associated with autism over the first 2 years of life. Brain, 138, 2046-2058.

30. Fingher, N., Dinstein, I., Ben-Shachar, M., Haar, S., Dale, A.M., Eyler, L., Pierce, K. and Courchesne, E. (2017) Toddlers later diagnosed with autism exhibit multiple structural abnormalities in temporal corpus callosum fibers. Cortex, 97 , 291-305.

31. Vitriol, E.A. and Zheng, J.Q. (2012) Growth cone travel in space and time: the cellular ensemble of cytoskeleton, adhesion, and membrane. Neuron, 73, 1068-1081.

32. Myers, J.P., Santiago-Medina, M. and Gomez, T.M. (2011) Regulation of axonal outgrowth and pathfinding by integrin-ECM interactions. Dev. Neurobiol., 71, 901-923.

33. Hansen, S.M., Berezin, V. and Bock, E. (2008) Signaling mechanisms of neurite outgrowth induced by the cell adhesion molecules NCAM and N-cadherin. Cell. Mol. Life Sci., 65, 3809-3821.

34. Maness, P.F. and Schachner, M. (2007) Neural recognition molecules of the immunoglobulin superfamily: signaling transducers of axon guidance and neuronal migration. Nat. Neurosci., 10, 19-26.

35. Sakurai, T. (2012) The role of NrCAM in neural development and disorders-beyond a simple glue in the brain. Mol. Cell. Neurosci., 49, 351-363.

36. Denisenko-Nehrbass, N., Oguievetskaia, K., Goutebroze, L., Galvez, T., Yamakawa, H., Ohara, O., Carnaud, M. and Girault, J.A. (2003) Protein 4.1B associates with both Caspr/paranodin and Caspr2 at paranodes and juxtaparanodes of myelinated fibres. Eur. J. Neurosci., 17, 411-416.

37. Gennarini, G., Bizzoca, A., Picocci, S., Puzzo, D., Corsi, P. and Furley, A.J.W. (2017) The role of Gpi-anchored axonal glycoproteins in neural development and neurological disorders. Mol. Cell. Neurosci., 81, 49-63.

38. Denisenko-Nehrbass, N., Goutebroze, L., Galvez, T., Bonnon, C., Stankoff, B., Ezan, P., Giovannini, M., Faivre-Sarrailh, C. and Girault, J.A. (2003) Association of Caspr/paranodin with tumour suppressor schwannomin/merlin and beta1 integrin in the central nervous system. J. Neurochem., 84, 209-221.

39. Pollock, N.S., Atkinson-Leadbeater, K., Johnston, J., Larouche, M., Wildering, W.C. and McFarlane, S. (2005) Voltage-gated potassium channels regulate the response of retinal growth cones to axon extension and guidance cues. Eur. J. Neurosci., 22, 569-578.

40. McFarlane, S. and Pollock, N.S. (2000) A role for voltage-gated potassium channels in the outgrowth of retinal axons in the developing visual system. J. Neurosci., 20, 1020-1029.

41. Klingler, E., Martin, P.M., Garcia, M., Moreau-Fauvarque, C., Falk, J., Chareyre, F., Giovannini, M., Chedotal, A., Girault, J.A. and Goutebroze, L. (2015) The cytoskeleton-associated protein SCHIP1 is involved in axon guidance, and is required for piriform cortex and anterior commissure development. Development, 142, 2026-2036. 